\title{
An Adaptive Multi-State Estimation Algorithm for Lithium-ion Batteries Incorporating Temperature Compensation
}

\author{
Xing Shu ${ }^{1}$, Guang $\mathrm{Li}^{2}$, Jiangwei Shen ${ }^{1}$, Zhenzhen $\mathrm{Lei}^{3}$, Zheng Chen ${ }^{1,2^{*}}$ and Yonggang $\mathrm{Liu}^{4 *}$ \\ ${ }^{1}$ Faculty of Transportation Engineering, Kunming University of Science and Technology, Kunming, 650500, China \\ ${ }^{2}$ School of Engineering and Materials Science, Queen Mary University of London, London, E1 4NS, United Kingdom \\ ${ }^{3}$ School of Mechanical and Power Engineering, Chongqing University of Science \& Technology, Chongqing, 401331, \\ China \\ ${ }^{4}$ State Key Laboratory of Mechanical Transmissions \& School of Automotive Engineering, Chongqing University, \\ Chongqing, 400044, China \\ Email: shuxing92@kust.edu.cn, g.li@qmul.ac.uk, shenjiangwei6@163.com,2010048@cqust.edu.cn, chen@kust.edu.cn, \\ andylyg@umich.edu \\ Corresponding Author: Zheng Chen (chen@kust.edu.cn) and Yonggang Liu (andylyg@umich.edu)
}

\begin{abstract}
Accurate estimation of inner status is vital for safe reliable operation of lithium-ion batteries. In this study, a temperature compensation based adaptive algorithm is proposed to simultaneously estimate the multi-state of lithium-ion batteries including state of charge, state of health and state of power. In the proposed co-estimation algorithm, the state of health is identified by the open circuit voltage-based feature point method. On the basis of accurate capacity prediction, the state of charge is estimated by the adaptive extended Kalman filter with a forgetting factor considering temperature correction. The state of power is determined according to the multi constraints subject to state of charge, operating temperature and maximum current duration. The substantial experimental validations in terms of different current profiles, aging status and time-varying temperature operating conditions highlight that the proposed algorithm furnishes preferable estimation precision with certain robustness, compared with the traditional extended Kalman filter and the adaptive extended Kalman filter. Moreover, the battery pack validation is performed to further justify the feasibility of proposed algorithm when employed in a product battery management system.
\end{abstract}

Key Words: Adaptive extended Kalman filter; state of charge; state of health; state of power; temperature compensation.

\section{NOMENCLATURE}

\section{Abbreviations}

EVs

electric vehicles

ECM

equivalent circuit model

BMS

$\mathrm{RC}$ resistance-capacitance

SOC state of charge

LiNMC lithium nickel-manganese-cobalt oxide 


\begin{tabular}{|c|c|c|c|}
\hline $\mathrm{SOH}$ & state of health & $\mathrm{OCV}$ & open circuit voltage \\
\hline SOP & state of power & MSE & mean squared error \\
\hline $\mathrm{Ah}$ & Ampere-hour & DOD & depth of discharge \\
\hline $\mathrm{OCV}$ & open circuit voltage & HPPC & $\begin{array}{l}\text { hybrid pulse power } \\
\text { characterization }\end{array}$ \\
\hline $\mathrm{KF}$ & Kalman filter & AEKF-FF & $\begin{array}{l}\text { adaptive extended Kalman filter } \\
\text { with a forgetting factor }\end{array}$ \\
\hline EKF & extended Kalman filter & PSO & particle swarm optimization \\
\hline AEKF & adaptive extended Kalman filter & RLS-FF & $\begin{array}{l}\text { recursive least square with } \\
\text { forgetting factor }\end{array}$ \\
\hline UKF & unscented Kalman filter & UDDS & $\begin{array}{l}\text { urban dynamometer driving } \\
\text { schedule }\end{array}$ \\
\hline $\mathrm{CKF}$ & cubature Kalman filter & $\mathrm{ME}$ & mean error \\
\hline RLS & recursive least square & RMSE & root mean square error \\
\hline ASRSPKF & $\begin{array}{l}\text { adaptive square root sigma-point Kalman } \\
\text { filter }\end{array}$ & $\mathrm{CC}$ & constant current \\
\hline SVM & support vector machine & DST & dynamic stress test \\
\hline NN & neural network & FUDS & federal urban driving schedule \\
\hline ELM & extreme learning machine & $\mathrm{CC}-\mathrm{CV}$ & constant current - constant voltage \\
\hline LSTM & long short-term memory network & $\mathrm{DC}$ & direct current \\
\hline PF & particle filter & $\mathrm{CAN}$ & controller area network \\
\hline LS-SVM & least squares support vector machine & & \\
\hline \multicolumn{4}{|l|}{ Symbols } \\
\hline$i$ & loading current & $B_{k}$ & control matrix \\
\hline$v_{t}$ & terminal voltage & $O C V_{\text {Mean }, S O H}$ & $\begin{array}{l}\text { mean of OCV at different } \mathrm{SOH} \\
\text { levels }\end{array}$ \\
\hline$v_{o c v}$ & open circuit voltage & $v_{\text {Feature }}$ & feature point voltage \\
\hline$R_{j}$ & resistor & $Q_{\text {Cum }}$ & cumulative discharging capacity \\
\hline$C_{q}$ & polarization capacitor & $S_{\text {Rest }}$ & look-up table SOC \\
\hline$s$ & state of charge & $Q_{\text {Init }}$ & rated capacity \\
\hline$T$ & battery temperature & $\varepsilon$ & relative error of current sensor \\
\hline$T_{s}$ & sampling interval & $\Delta$ & $\begin{array}{l}\text { the maximum sampling error of } \\
\text { cell voltage }\end{array}$ \\
\hline$\eta$ & denotes the coulomb efficiency & $f\left(v_{\text {Rest }}\right)$ & true SOC \\
\hline$Q_{a}$ & battery available capacity & $f\left(v_{\text {Rest }}+\Delta\right)$ & $\begin{array}{l}\text { measurement SOC according to } \\
\text { the look-up table }\end{array}$ \\
\hline$s(k)$ & the state of charge at the $k$ th sampling step & $Q_{\text {Cum_True }}$ & $\begin{array}{l}\text { true cumulative discharging } \\
\text { capacity }\end{array}$ \\
\hline$p_{j}$ & fitting coefficients of OCV & $Q_{\text {Cum_Mea }}$ & $\begin{array}{l}\text { measurement cumulative } \\
\text { discharging capacity }\end{array}$ \\
\hline$v_{\text {Mea }}$ & measured terminal voltage & $Q_{a_{-} \text {True }}$ & true available capacity \\
\hline
\end{tabular}




\begin{tabular}{|c|c|c|c|}
\hline$N$ & total sampling time & $Q_{a_{-} \text {Mea }}$ & measured available capacity \\
\hline$E_{p, k}$ & historical error innovation & $t_{\text {Dis__True }}$ & true discharging time \\
\hline$p$ & window size of innovation update & $t_{\text {Dis_-Mea }}$ & measured discharging time \\
\hline$e_{k}$ & error matrix at the $k$ th sampling step & mean $(\cdot)$ & mean value \\
\hline$K_{k}$ & Kalman gain & $\mathfrak{R}$ & the first-order residual \\
\hline$\hat{x}_{k}^{-}$ & priori state matrix & $S_{\min }$ & lower SOC value \\
\hline$\hat{x}_{k}^{+}$ & posterior state matrix & $S_{\max }$ & upper SOC value \\
\hline$\lambda$ & forgetting factor & $i_{k}^{d i s, s}$ & $\begin{array}{l}\text { discharge current capability } \\
\text { limited by SOC }\end{array}$ \\
\hline$I$ & unit matrix & $i_{k}^{c h a, s}$ & $\begin{array}{l}\text { charge current capability limited } \\
\text { by SOC }\end{array}$ \\
\hline$Q_{k}$ & process noise matrix & $i_{k}^{d i s, v}$ & $\begin{array}{l}\text { discharge current capability } \\
\text { limited by voltage }\end{array}$ \\
\hline$P_{k}^{-}$ & prior error covariance matrix & $i_{k}^{c h a, v}$ & $\begin{array}{l}\text { charge current capability limited } \\
\text { by voltage }\end{array}$ \\
\hline$R_{k}$ & measurement noise covariance matrix & $i^{d i s, m}$ & $\begin{array}{l}\text { continuous discharge current } \\
\text { capability of its design limit }\end{array}$ \\
\hline$P_{k}^{+}$ & posterior error covariance matrix & $i^{\text {cha,m }}$ & $\begin{array}{l}\text { continuous charge current } \\
\text { capability of its design limit }\end{array}$ \\
\hline$H_{k}$ & variance-covariance matrix & $\hat{i}_{k+l}^{d i s}$ & $\begin{array}{l}\text { multi-constraint discharge current } \\
\text { capability }\end{array}$ \\
\hline$M$ & size of moving window & $\hat{i}_{k+l}^{c h a}$ & $\begin{array}{l}\text { multi-constraint charge current } \\
\text { capability }\end{array}$ \\
\hline$u_{k}$ & input variable & $P_{k+l}^{d i s}$ & multi-constraint discharge SOP \\
\hline$A_{k}$ & system matrix & $P_{k+l}^{c h a}$ & multi-constraint charge SOP \\
\hline
\end{tabular}

\section{INTRODUCTION}

Lithium-ion batteries have been progressively deployed in electric vehicles (EVs) and energy storage systems because of their long cycle life and high energy density [1]. To guarantee proper safe operation of batteries, their management systems (BMSs) emerge to conduct essential tasks including signal monitoring, inner state estimation, charge and thermal control as well as communication [2]. An important function of BMS is to timely estimate all-functional inner status, such as state of charge (SOC), state of health (SOH) and state of power (SOP) [3], which has been paid much attention by industry and academia.

\section{A. SOC Estimation}

SOC denotes the percentage of current available capacity over the rated value and is mostly concerned by users and manufacturers. It can supply the reference of how long the battery can be fully charged and how much energy is left inside of the battery. Generally, SOC estimation algorithms can be simply divided into closed-loop methods and open-loop methods [4]. The Ampere-hour (Ah) integration method (also known as 
Ah counting or coulomb counting algorithm) and open circuit voltage (OCV) look-up table method are two representatives of open-loop ones. Distinctly, they are simple and easy to implement, and most of product BMSs prefer to adopt the Ah integration method [5]. However, it is, as well known, susceptible to the current sampling accuracy and highly dependent on the initial value of SOC. A main concern is that when a fixed offset exists in the current sensor or transducer, the error will gradually accumulate with operation and finally lead to large discrepancy. While for the OCV look-up table method, adequate still duration discounts application potential in practice. Moreover, the voltage sampling accuracy also shows large influence on estimation accuracy of SOC.

To mitigate distinct drawbacks of open-loop methods, closed-loop SOC estimation methods are spurred. They can be divided into model based methods and learning based methods. Currently, model based methods are widely employed thanks to their satisfactory precision, effective noise cancellation, automatic convergence and easy implementation [6]. On the basis of built electrical or electro-chemical models, such as the equivalent circuit model (ECM) and pseudo single particle model, a number of algorithms have been extensively investigated and applied. The most prevailing manner for SOC estimation belongs to Kalman filter (KF) and its extension format, such as extended KF (EKF), adaptive EFK (AEKF), unscented KF (UKF) and cubature $\mathrm{KF}(\mathrm{CKF}) . \mathrm{KF}$ is effective in alleviating noises arisen during modeling and measurement [7]. In the conventional $\mathrm{KF}$, the optimal estimation can be achieved for linear systems with the help of accurate battery model; and for nonlinear systems, EKF is applied by approximating the system into the linear type at current step in an equivalent manner [8]. However, the amplitude of actual current noise is difficult, and sometimes even impossible, to acquire or estimate in a precise manner. Usually, the noise variance and covariance are simply regarded as a constant value, which reduces the estimation accuracy and the calculation process may even be divergent [9]. To cope with this deficiency, AEKF is introduced to update the covariance matrix iteratively, and by this manner, the noise covariance matrix gradually converges to the true value [10]. In [11], to alleviate the adverse effect of noise in the conventional recursive least square (RLS)-based parameter identification, the bias compensation RLS and EKF algorithms are presented for parameters recognition and SOC estimation in different noise corruption conditions. Ref. [12] employs a temperature dependent model to characterize the electrical behavior of lithium-ion batteries when the operating temperature ranges from $-10{ }^{\circ} \mathrm{C}$ to $40{ }^{\circ} \mathrm{C}$, and the parameters and $\mathrm{SOC}$ are estimated by the set 
membership theory, which supposes the system noise is unknown but bounded. In [13], on the basis of a simplified electrochemical model, the SOC is estimated by the adaptive square root sigma-point KF (ASRSPKF).

Learning-based algorithms treat the battery as a black box instead of a precise electrical or electrochemical model. They estimate battery SOC mainly through data statistics as well as nonlinear training and prediction. The typical algorithms include support vector machine (SVM) [14], neural networks (NNs) [15] and extreme learning machine (ELM) [16]. In the premise of enough prerequisite data and learning knowledge, these estimation methods can predict battery SOC with desired precision. Nevertheless, they are sensitive to quality of training data and selection of feature parameters [17]. In [18], the nonlinear relationship among SOC and measured current, voltage and temperature is mapped by the recurrent NN. Ref. [19] utilizes the long short-term memory network (LSTM) to forecast SOC, and the adaptive cubature Kalman filter is applied to smooth the output of LSTM. Besides, most of time, existing SOC estimation algorithms can attain better estimation performance in the case of fresh battery; and the estimation accuracy of SOC may deteriorate when the battery ages. Indeed, to ensure the estimation precision of SOC, the SOH should be estimated reliably to supply the reference of current capacity.

\section{B. SOH Estimation}

$\mathrm{SOH}$, indicating aging status of the battery, can be defined as percentages of current maximum capacity over the rated value [20]. Common estimation algorithms for $\mathrm{SOH}$ include direct measurement, model based methods and statistical methods [21]. The capacity test and internal resistance measurement are the most direct manners of obtaining battery $\mathrm{SOH}$ [22]. They can be easily implemented; nonetheless, the estimation precision cannot be guaranteed all the time, as the parameters may complicatedly vary with operating environments [17]. Model based methods, such as KF [23] and particle filter (PF) [24], can attain $\mathrm{SOH}$ estimation with preferable accuracy [25]; whereas their robustness cannot be guaranteed, and credibility of battery models require in-depth understanding of degradation mechanism [26]. The statistical methods, including Bayesian network [27], SVM [14], Gaussian process regression [20] and NN, can achieve SOH estimation by numerical analysis and without relying on early modeling process. For instance, Ref. [28] presents an application of least squares SVM (LS-SVM) in SOH prediction, where the grey relational analysis method is designed for feature extraction. In [29], to involve the input characteristics of temperature 
and depth of discharge (DOD), an improved kernel function is applied, and the inputs of the Gaussian process regression method is employed to obtain more accurate SOH estimation. A main concern is that they entail much effort to train the model, and furthermore data sufficiency needs to be fully addressed.

\section{SOP Estimation}

To ensure normal safe operation of lithium-ion batteries, SOP, as a key criterion, is a momentous parameter that needs to be precisely provided by BMS. Actually, it is difficult to give an authentic definition for SOP. Usually, SOP can be characterized by the maximum power in current stage, and apparently it is strongly related with SOC and SOH [30]. Presently, a variety of SOP estimation methods are explored, and existing representatives can be categorized into three groups, i.e. experiment based, voltage based, and SOC based [31]. The hybrid pulse power characterization (HPPC) test [32] is commonly performed to quantify the peak power under static conditions. Nevertheless, it cannot be well suited for dynamic operation and capacity degradation conditions. Voltage based methods, depending on accurate electrical models with high fidelity, are appropriate for SOP estimation and can ensure acceptable estimation accuracy [33]. However, these methods cannot attain high-quality SOP estimation all the time under different conditions due to lack of effective SOC and temperature feedback [34]. SOC based method limits the charge and discharge current by setting the allowable operating range of SOC [35]. From this point of view, the SOP estimation necessitates accurate SOC observation in turn. However, a body of researches reveal that when SOC locates within a wide range, the simple SOC based method may lead to outrageous SOP estimation that is beyond the battery's capability. To integrate the advantages of different SOP estimation methods, a multi-constraint based method is formed. In [36], given the SOC estimation by the Luenberger observer, the SOP is monitored online with consideration of the terminal voltage and SOC limitation. In [37], the RLS method is applied to identify model parameters, the EKF algorithm is developed to estimate SOC and the SOP is observed by a multi-constraint method. However, in their work, two different ECMs, second-order model and Rint model are respectively exploited in SOC and SOP prediction. Obviously, the computation intensity of BMS increases, and the oversimplified Rint model cannot capture the battery characteristics with enough precision. 


\section{Joint Estimation}

These mentioned approaches declare to estimate SOC, SOH or SOP individually; however, as discussed previously, these three states are essentially closely coupled with each other [37]. In this context, some researches have focused on their joint estimation. Popular solutions include a hierarchical framework for estimating SOC and SOH simultaneously; where the inner loop takes charge of SOC estimation, and the outer loop accounts for SOH estimation [38]. In [39], the Elman neural network is leveraged to predict the battery capacity, and AEKF is employed to achieve the SOC estimation with timely updated battery model and parameters. In [40], a novel estimation of remaining energy and SOC of battery pack at different temperatures are cooperatively estimated by UKF. In [41], a simple co-estimation method is proposed for simultaneous SOC and current capacity prediction by extracting three feature variables from incremental capacity profiles and differential voltage curves. In [42], an improved moving horizon estimation is firstly introduced to estimate the SOC online by considering the convergence and fault tolerance. Then, the Ah integration and the estimated SOC are synthetically referred to obtain the capacity offline. Finally, the updated model parameters and estimated states are integrated to predict SOP. However, existing literature still show some distinct deficiencies that need to be properly tackled before implementation in EV. First, the effect of temperature on estimation of inner states is insufficiently considered, as most of the reported methods in the literature adopt ideal working environment temperature, even some researches have focused on performance variation under different temperature conditions. Nonetheless, three parameters' joint estimation is seldom reported under the whole operating temperature range, such as high temperature of more than $50{ }^{\circ} \mathrm{C}$. Second, the aforementioned single or joint estimation is conducted in restricted conditions, and real-time joint estimation accuracy of inner states $\mathrm{o}$ in the real environment still needs further exploration. Additionally, the computation intensity should also be properly taken into account when applied in engineering practice.

Motivated by the discussed challenges, a multi-state co-estimation framework for SOC, SOH and SOP is herein constructed to satisfy demand of real-time implementation and cope with wide operating temperature range. To this end, an effective electrical model is built with the consideration of influences arisen by temperature variation. Based on the identified model parameters at different temperatures, the AEKF with a forgetting factor (called AEKF-FF hereinafter) is formulated to achieve the SOC estimation 
with temperature compensation and the $\mathrm{SOH}$ is estimated by means of a simple OCV based feature point method. In addition, the SOP is estimated in line with the constraints formulated by existing temperature, current, voltage and SOC constraints. The built algorithm has been validated in a product BMS to estimate the states of a battery module which includes fourteen cells connected in series topology. The experimental results indicate the multi-state estimation feasibility of proposed algorithm in reducing the influence incurred by different aging states and operating temperature variation. The main contributions of this study can be attributed to the following two aspects: 1) A comprehensive battery model accounting for the operating temperature range of $-20{ }^{\circ} \mathrm{C}$ to $60{ }^{\circ} \mathrm{C}$ is established to efficaciously find the available capacity and OCVSOC relationship with respect to different temperature; 2) An improved AEKF algorithm with a forgetting factor is developed to achieve accurate and reliable SOC estimation by fully considering the temperature variation, and on this basis, accurate estimation of inner states of batteries at high temperature and timevarying temperature are achieved.

The remainder of this paper is structured as follows: Section II models the lithium-ion battery and identifies the model parameters. The framework of co-estimator is discussed in Section III. In Section IV, the off-line comprehensive experiments are conducted to verify the proposed co-estimation algorithm. In Section $\mathrm{V}$, the validation in a product BMS is conducted and the feasibility of proposed algorithm is fully verified. Finally, the main conclusions are given in Section VI.

\section{MODEL ANALYSIS AND PARAMETERS IDENTIFICATION}

To apply model-based methods to estimate battery inner states, a preliminary task is to establish an effective model with acceptable precision and calculation labor.

\section{A. Lithium-ion Battery Model}

We adopted an equivalent circuit model (ECM), as shown in Fig. 1 (a), to characterize the battery's electrical performance thanks to its capability of fast calculation and preferable accuracy. The parameters of studied lithium nickel-manganese-cobalt oxide (LiNMC) battery are specified in Table I. To better consider the polarization characteristics of battery, a two-order resistance-capacitance (RC) network together with a 
serial connected resistor and an OCV source is applied to simulate the static and dynamic electrical performance [43].

Table I. Specification of cells.

\begin{tabular}{ll}
\hline Items & Specifications \\
\hline Cathode material & $\mathrm{Li}(\mathrm{NiCoMn}) \mathrm{O}_{2}$ \\
Anode material & Graphite \\
Nominal capacity & $4 \mathrm{Ah}$ \\
Allowed operating range of voltage & $2.75-4.2 \mathrm{~V}$ \\
Rated voltage & $3.65 \mathrm{~V}$ \\
Allowable charging temperature & $0-45{ }^{\circ} \mathrm{C}$ \\
Allowable discharging temperature & $-20-60^{\circ} \mathrm{C}$ \\
\hline
\end{tabular}

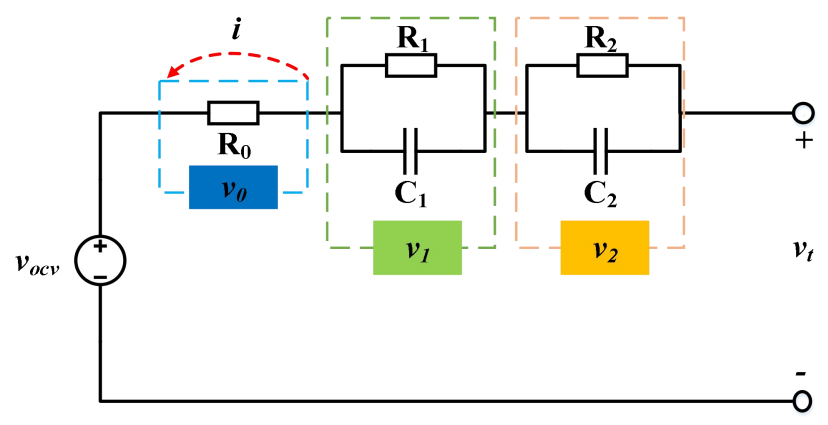

(a)
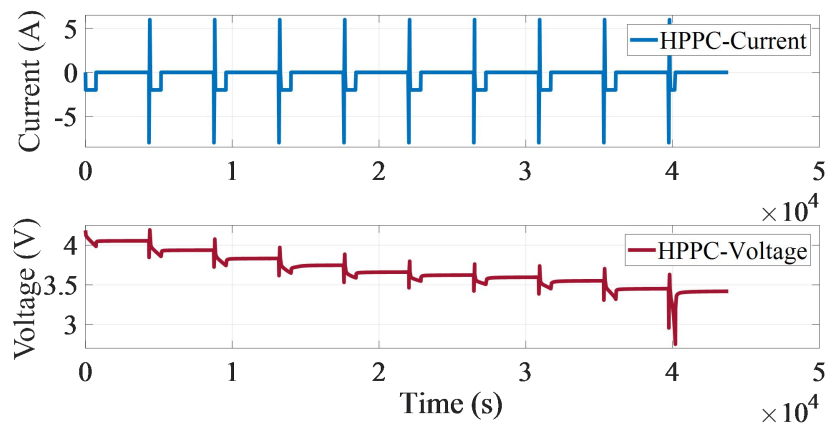

(b)

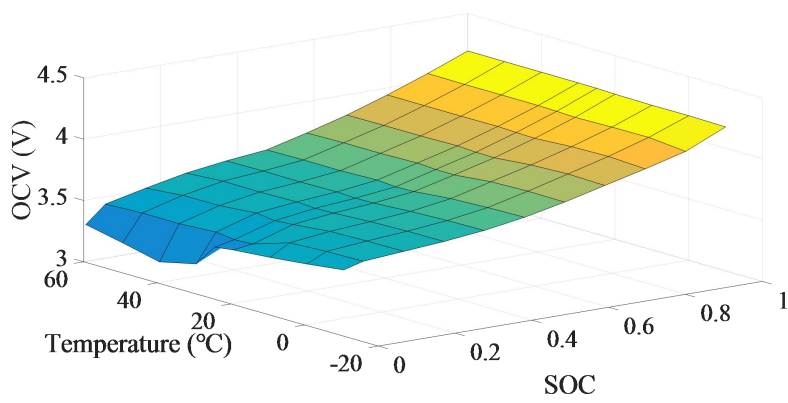

(c)

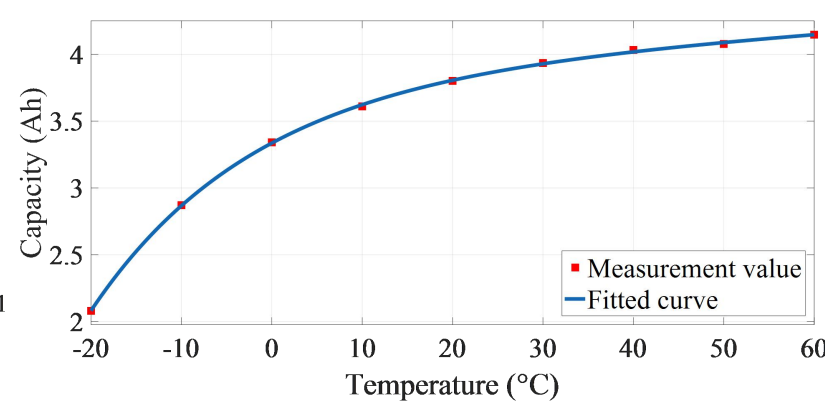

(d)

Fig. 1. Battery model and parameters. (a) Schematic diagram of battery model; (b) HPPC test curves at $25^{\circ} \mathrm{C}$; (c) OCV-SOC-T relationship; (d) Available capacity profile. $i$ and $v_{t}$ are the battery current and terminal voltage. $v_{o c v}$ denotes the battery OCV. $v_{j}, j=0,1,2$ is the voltage across resistor $R_{j}$, and $C_{q}, q=1,2$ is the polarization capacitor.

As can be seen in Fig. 1 (a), the OCV is used to describe the battery static terminal voltage, and it is strongly related with SOC, temperature and degradation status, as: 


$$
v_{o c v}=f_{o c v}(s, S O H, T)
$$

where $s$ denotes the battery SOC, $T$ represents the battery temperature, and the relationship will be discussed in the next section. The voltage across the internal resistor and RC network can be formulated, as:

$$
\left\{\begin{array}{l}
v_{j}(k+1)=a_{j} v_{j}(k)+b_{j} i(k), j=1,2 \\
v_{0}(k+1)=R_{0} i(k)
\end{array}\right.
$$

where $a_{j}=e^{-T_{s} / \tau_{1}}, b_{j}=R_{j}\left(1-a_{j}\right)$, and $T_{s}$ denotes the sampling interval. The battery SOC can be calculated by the Ah integration method, as:

$$
s(k+1)=s(k)+\frac{\eta T_{s}}{Q_{a}} i(k)
$$

where $\eta$ denotes the coulomb efficiency, $Q_{a}$ denotes the battery available capacity and is related to the operating temperature and SOH. $s(k)$ stands for the SOC at the $k$ th sampling step. According to Fig. 1 (a), the estimated voltage can be derived, as:

$$
v_{t}(k)=v_{o c v}(k)+v_{0}(k)+v_{1}(k)+v_{2}(k)
$$

Next, the model parameters will be identified.

\section{B. Parameters Identification}

In this paper, the HPPC test, which can be referred in our previous research in [44], are conducted under different temperatures for identification of model parameters. The current and voltage profiles at $25{ }^{\circ} \mathrm{C}$ are depicted in Fig. 1 (b). During the HPPC test, the voltage after shelved for 1 hour is considered as the OCV. By repeating the experiments under different temperatures, a three-dimensional table can be built to depict the nonlinear relationship among OCV, temperature and SOC, as plotted in Fig. 1 (c). The available capacity at different temperatures are sketched in Fig. 1 (d). When the temperature rises, the available capacity increases accordingly. Here, a polynomial function is employed to fit the relationship between temperature and available capacity, as:

$$
Q_{a}=p_{1} \exp \left(p_{2} \cdot T\right)+p_{3} \exp \left(p_{4} \cdot T\right)
$$

where $p_{j}, j=1,2,3,4$ denotes the fitting coefficients. $p_{1}=3.913, p_{2}=0.001051, p_{3}=-0.5771$, and $p_{3}=-0.05546$. According to (5), the available capacity values at $-20{ }^{\circ} \mathrm{C}$ to $60{ }^{\circ} \mathrm{C}$ can be easily obtained. The identification of $R_{0}, R_{1}, R_{2}, C_{1}$ and $C_{2}$ can be considered as an optimization problem [45], of which 
the target function is defined as minimization of the mean squared error (MSE) between the measured terminal voltage and model output, as:

$$
\min M S E=\frac{1}{N} \sum_{k=1}^{N}\left(v_{t}(k)-v_{\text {Mea }}(k)\right)^{2}
$$

where $v_{\text {Mea }}$ denotes the measured terminal voltage, and $N$ is the total sampling time. In this study, the particle swarm optimization (PSO) algorithm is employed to solve the optimization problem own to its advantages of less parameters, simpler theory and satisfactory searching performance.

In the next step, the joint algorithm is designed to achieve the inner states estimation of battery.

\section{Design Of THE Co-Estimation AlgoRithm}

The framework of joint status estimation proposed in this paper is sketched in Fig. 2. As can be observed, it mainly consists of four parts: determination of model parameters, SOH estimation, SOC estimation and SOP estimation. The main task of model parameter module is to determine appropriate parameters according to different operating temperature. The $\mathrm{SOH}$ estimation module is in charge of calculating the battery capacity and helping estimation of SOC and SOP. The calculated SOH and selected parameters are then fed back to the AEKF-FF to estimate the battery SOC, as seen in the SOC estimation module. Simultaneously, the SOP module takes charge of predicting the maximum charge/discharge power by considering the limitations of voltage, current, SOC and incorporating the selected model parameters, the estimated SOC and $\mathrm{SOH}$. The detailed co-estimation procedures are elaborated as follows. 


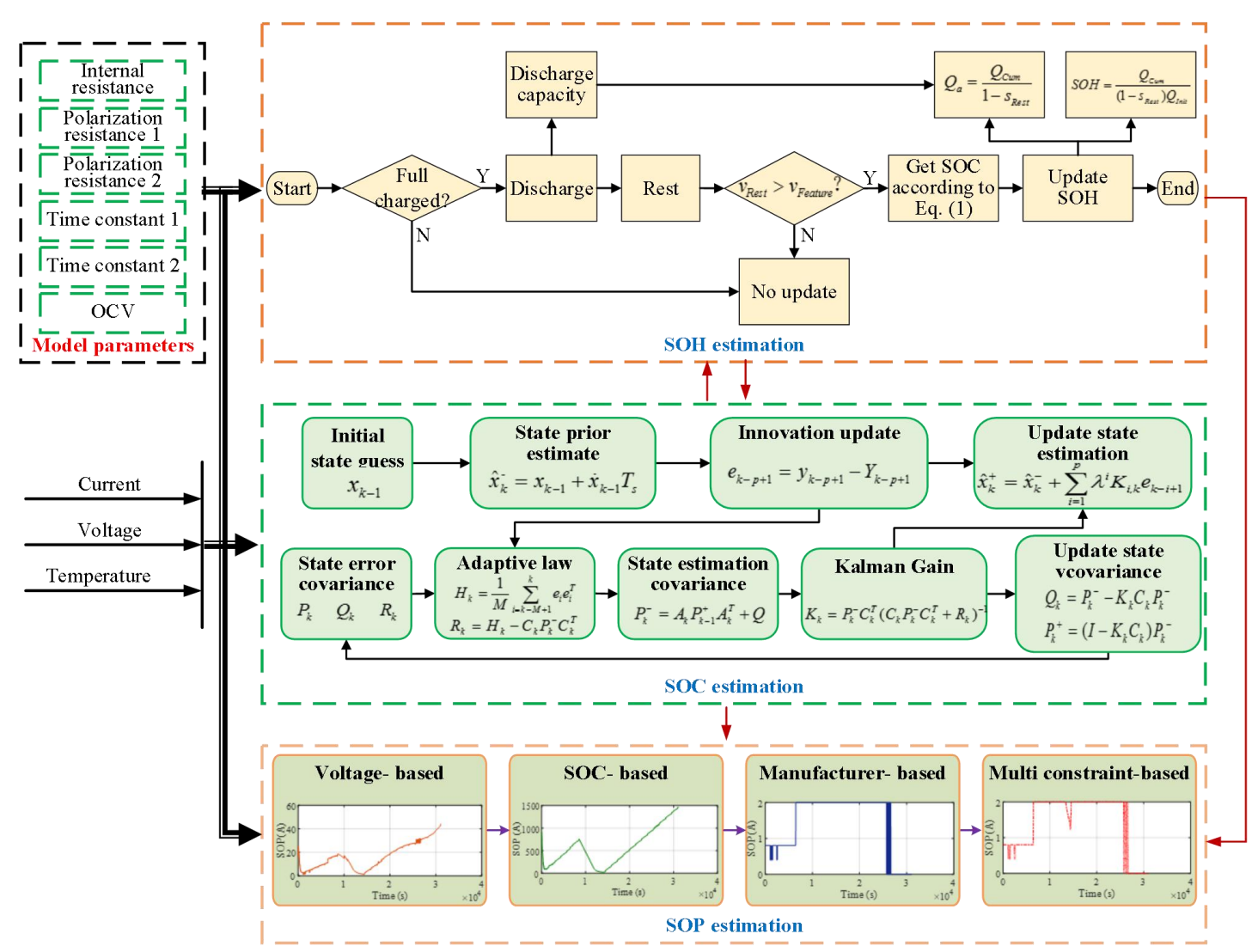

Fig. 2. The joint estimation framework.

\section{A. SOC Estimation}

In this study, the AEKF-FF is exerted to estimate the battery SOC. For a traditional AEKF, only the current error is regarded as the state estimate measurement update, which may result in loss of information for posterior measurement correction. Inspired by the RLS with forgetting factor (RLS-FF) [46], an updated AEKF algorithm with a forgetting factor is introduced to improve the error correction effect. The historical error update $E_{p, k}$ can be expressed as:

$$
E_{p, k}=\left[\begin{array}{c}
e_{k} \\
e_{k-1} \\
\vdots \\
e_{k-p+1}
\end{array}\right]=\left[\begin{array}{c}
y_{k}-g\left(\hat{x}_{k}^{-}, u_{k}\right) \\
y_{k-1}-g\left(\hat{x}_{k-1}^{-}, u_{k-1}\right) \\
\vdots \\
y_{k-p+1}-g\left(\hat{x}_{k-p+1}^{-}, u_{k-p+1}\right)
\end{array}\right]
$$

where $p$ denotes the window size of update. Meanwhile, the traditional Kalman gain $K_{k}$ can be extended, as:

$$
K_{p, k}=\left[K_{k}, K_{k-1}, \cdots, K_{k-p+1}\right]
$$

The state update can be obtained, as:

$$
\hat{x}_{k}^{+}=\hat{x}_{k}^{-}+K_{p, k} E_{p, k}=\hat{x}_{k}^{-}+\sum_{i=1}^{p} K_{k-i+1} e_{k-i+1}
$$


where $\hat{x}_{k}^{-}$and $\hat{x}_{k}^{+}$denote the priori and posteriori estimation, respectively. Note that when $p=1$, the proposed AEKF-FF degrades to the traditional AEKF. From (9), we can find in the update window, the historical information contributes mostly to the state update, thus hindering the newly referred data from correction. Meanwhile, the measurement and estimation error of historical data may lead to cumulative interference, thereby weakening the filtering effect and affecting the SOC estimation precision. In this case, a forgetting factor is introduced to reduce the accumulation effect of historical data. The update of measurement with the forgetting factor is detailed as:

$$
\hat{x}_{k}^{+}=\hat{x}_{k}^{-}+K_{p, k} E_{p, k}=\hat{x}_{k}^{-}+\sum_{i=1}^{p} \lambda^{i} K_{i, k} e_{k-i+1}
$$

where $\lambda$ denotes the forgetting factor. Here, we assume that the weight sum of historical data contributes the same effect to innovation update, and $\lambda$ is set to 0.5 . Based on the built ECM, the implementation process of improved AEKF-FF can be formulated, as:

$$
\left\{\begin{array}{l}
\hat{x}_{k}^{-}=A_{k} \hat{x}_{k-1}^{+}+B_{k} u_{k} \\
P_{k}^{-}=A_{k} P_{k-1}^{+} A_{k}^{T}+Q_{k} \\
e_{k-p+1}=y_{k-p+1}-C_{k-p+1} \hat{x}_{k-p+1}^{-}-D_{k-p+1} u_{k-p+1} \\
H_{k}=\frac{1}{M} \sum_{i=k-M+1}^{k} e_{i} e_{i}^{T}, R_{k}=H_{k}-C_{k} P_{k}^{-} C_{k}^{T} \\
P_{k}^{+}=\left(I-K_{k} C_{k}\right) P_{k}^{-} \\
K_{k}=P_{k}^{-} C_{k}^{T}\left(C_{k} P_{k}^{-} C_{k}^{T}+R_{k}\right)^{-1} \\
Q_{k}=P_{k}^{-}-K_{k} C_{k} P_{k}^{-} \\
\hat{x}_{k}^{+}=\hat{x}_{k}^{-}+\sum_{i=1}^{p} \lambda^{i} K_{i, k} e_{k-i+1}
\end{array}\right.
$$

where $I$ is the unit matrix, $K_{k}$ is the Kalman gain matrix, $\hat{x}_{k}^{+}$is the posterior state matrix, $e_{k}$ denotes the error matrix, $Q_{k}$ is the process noise matrix, $P_{k}^{-}$is the prior error covariance matrix, $R_{k}$ denotes the measurement noise covariance matrix, $\hat{x}_{k}^{-}$is the prior state matrix, $P_{k}^{+}$is the posterior error covariance matrix, $H_{k}$ is the variance-covariance matrix, and $M$ is the size of moving window. Additionally, $u_{k}, A_{k}$, $B_{k}, C_{k}$ and $D_{k}$ are respectively defined as: 


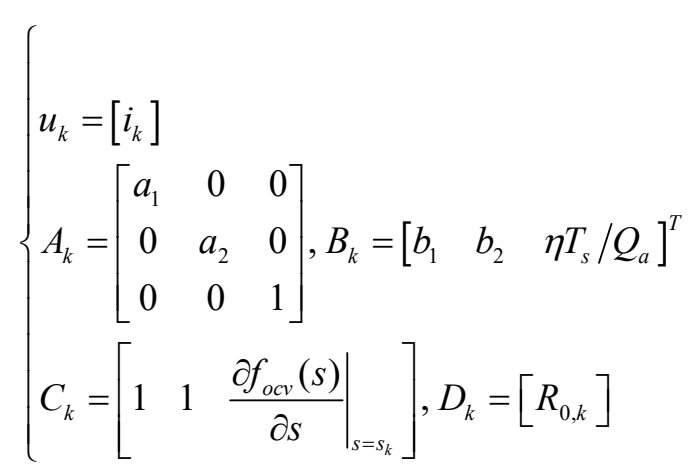

\section{B. SOH Estimation}

Fig. 3 (a) and (b) sketch the relationship among OCV, SOC, SOH and temperature. It is obvious that the SOC-OCV profile remains almost the same in the whole lifespan of battery and only drifts slightly when the SOC is lower than $60 \%$. Thus, we can conclude that a relatively deterministic mapping function exists between the OCV and SOC, and instead weak relationship is identified between the OCV and $\mathrm{SOH}$, as explained in [47]. Thus, the OCV based look-up table can be referred to help the SOC estimation based on the Ah integration method [37]. The mean value of OCV at different $\mathrm{SOH}$ is calculated to diminish the impact of battery service life on OCV estimation, as expressed in (13) and plotted in Fig. 3 (c) and (d). As can be observed, the OCV is independent of $\mathrm{SOH}$, and the maximum OCV difference with the same SOC and different $\mathrm{SOH}(80 \%$ to $100 \%)$ is less than $0.005 \mathrm{~V}$ when the battery SOC higher than $62.5 \%$.

$$
O C V_{\text {Mean }, \text { SOH }}=\operatorname{mean}\left(O C V_{100 \% \mathrm{SOH}}, O C V_{90 \% \mathrm{SOH}}, O C V_{80 \% \mathrm{SOH}}\right)
$$

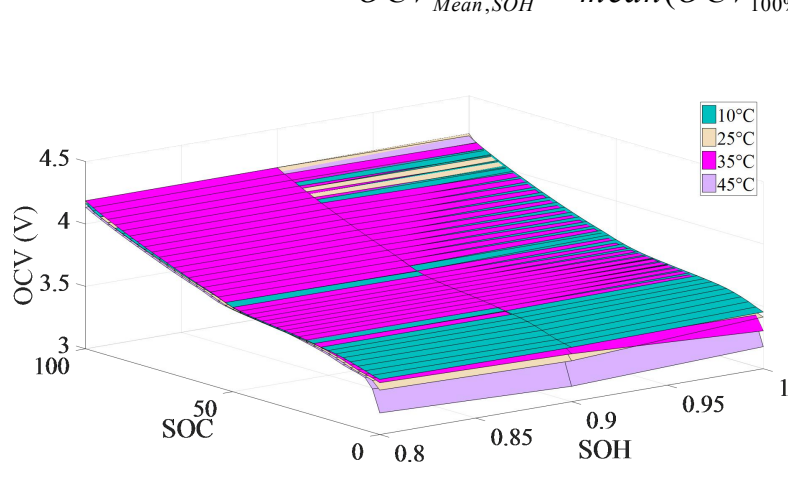

(a)

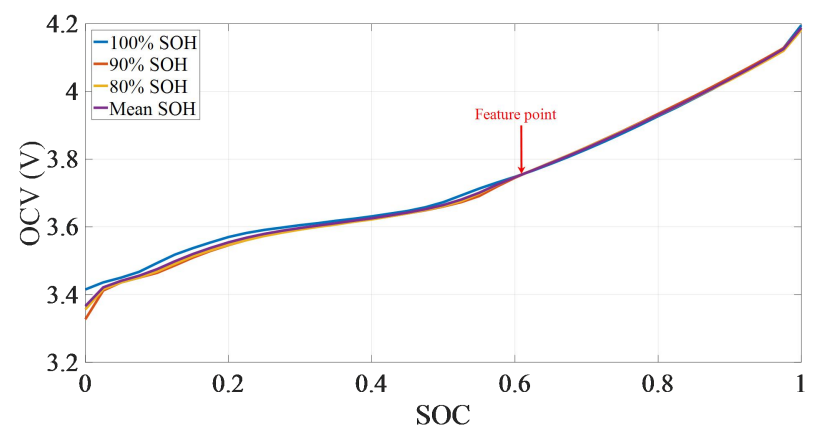

(c)

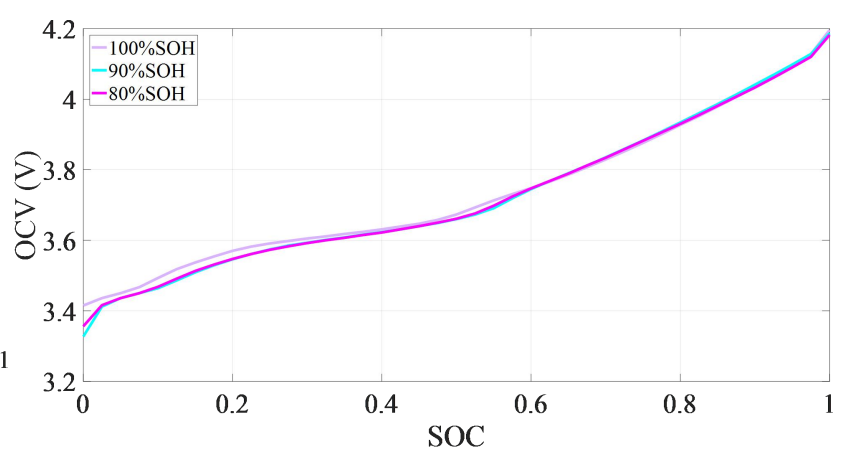

(b)

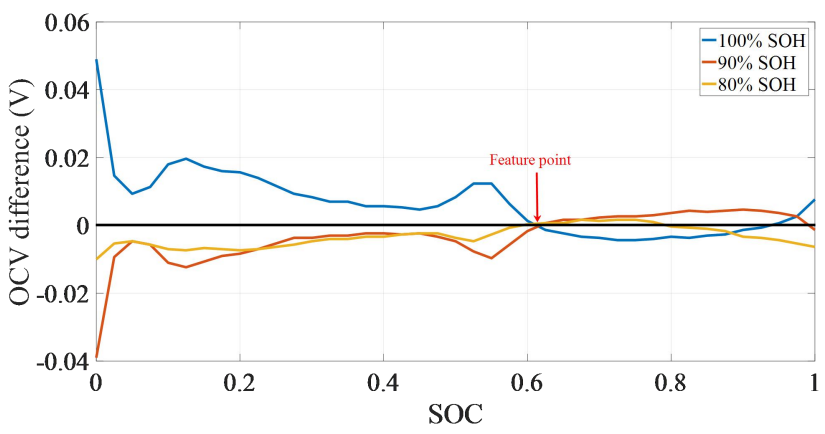

(d) 
Fig. 3. The relationship among OCV, SOH, SOC and temperature. (a) OCV 3-D response surface; (b) OCV at different SOH; (c) Mean value of OCV; (d) OCV difference.

Thus, the $\mathrm{SOH}$ can be calculated by accumulating the capacity within certain range. The proposed operating processes of SOH estimation can be summarized as follows:

Step 1: Fully charge the battery.

Step 2: Calculate the cumulative discharging capacity when the battery is in operation.

Step 3: When the battery is adequately shelved and the terminal voltage after rest is higher than the feature point voltage $\left(v_{\text {Feature }}=3.7673 \mathrm{~V}\right.$ corresponding to the SOC of $\left.62.5 \%\right)$, go to next step. Otherwise, terminate the process without updating the $\mathrm{SOH}$.

Step 4: Obtain the current SOC by looking up the mean value of OCV.

Step 5: Calculate the available capacity as:

$$
Q_{a}=\frac{Q_{\text {Cum }}}{1-s_{\text {Rest }}}
$$

where $Q_{\text {Cum }}$ is the cumulative discharging capacity, $s_{\text {Rest }}$ denotes the SOC obtained by the look-up table. Now, the battery SOH can be derived as:

$$
S O H=\frac{Q_{\text {Cum }}}{\left(1-s_{\text {Rest }}\right) Q_{\text {Init }}}
$$

where $Q_{\text {Init }}$ denotes the rated capacity. It is worth noting that during the battery service life, the $\mathrm{SOH}$ update conditions are not satisfied in each operation; whereas, it is not necessary to estimate the $\mathrm{SOH}$ in each operation, as the battery capacity declines very slowly and usually does not change obviously in a short period [48]. In order to illustrate the capacity estimation error based on the mathematical derivation, we suppose that the discharging current is $i_{k}$ at step $k$, the voltage after rest is $v_{\text {Rest }}$, the relative error of current sensor is $\varepsilon$, the maximum sampling error of cell voltage is $\Delta$, the real SOC and calculated SOC based on the look-up table are $f\left(v_{\text {Rest }}\right)$ and $f\left(v_{\text {Rest }}+\Delta\right)$, respectively. Thus, the discharged capacity can be calculated, as:

$$
\left\{\begin{array}{l}
Q_{\text {Cum_True }}=\left(1-f_{\text {ocv }}\left(v_{\text {Rest }}\right)\right) Q_{a_{-} \text {Ture }} \\
Q_{\text {Cum_Mea }}=\left(1-f_{\text {ocv }}\left(v_{\text {Rest }}+\Delta\right)\right) Q_{a_{-} \text {Mea }}
\end{array}\right.
$$


where $Q_{\text {Cum_true }}$ and $Q_{\text {Cum_Mea }}$ express the cumulative discharging capacity and measurement cumulative discharging capacity, respectively. $Q_{a_{-} \text {True }}$ denotes the available capacity, and $Q_{a_{-} \text {Mea }}$ represents the measured available capacity. Given this, the total discharge time can be calculated, as:

$$
\left\{\begin{array}{l}
t_{\text {Dis_True }}=\frac{\left(1-f_{\text {ocv }}\left(v_{\text {Rest }}\right)\right) Q_{a_{-} \text {Ture }}}{\text { mean }(i)} \\
t_{\text {Dis_Mea }}=\frac{\left(1-f_{\text {ocv }}\left(v_{\text {Rest }}+\Delta\right)\right) Q_{a_{-} \text {Mea }}}{\text { mean }(i(1+\varepsilon))}
\end{array}\right.
$$

where $t_{\text {Dis_True }}$ and $t_{\text {Dis_Mea }}$ express the true discharging time and measured discharging time, respectively. mean $(\cdot)$ denotes the mean value. Owing to $t_{\text {Dis__True }}=t_{\text {Dis_Mea }}$, the available capacity can be attained, as:

$$
Q_{a_{-} \text {Mea }}=\frac{\frac{\left(1-f_{\text {ocv }}\left(v_{\text {Rest }}\right)\right) Q_{a}}{\text { mean }(i)} \text { mean }(i(1+\varepsilon))}{\left(1-f_{\text {ocv }}\left(v_{\text {Rest }}+\Delta\right)\right)}
$$

Finally, the difference between the real capacity and calculated capacity can be determined considering the error of current sensor and monitoring precision, as:

$$
\begin{aligned}
R E_{\text {Capcity }}= & \frac{\left(1-f_{\text {ocv }}\left(v_{\text {Rest }}\right)\right) Q_{a_{-} \text {True }}}{\text { mean }(i)} \text { mean }(i(1+\varepsilon)) \\
\left(1-f_{\text {ocv }}\left(v_{\text {Rest }}+\Delta\right)\right) & Q_{a_{-} \text {True }} \\
= & \frac{\left(1-f_{\text {ocv }}\left(v_{\text {Rest }}\right)\right)(1+\varepsilon)}{\left(1-f_{\text {ocv }}\left(v_{\text {Rest }}+\Delta\right)\right)}-1
\end{aligned}
$$

From (19), we can conclude that the SOH difference between the real capacity and calculated capacity depends on the slope of OCV-SOC curve and the error of current and voltage sensors. Undoubtedly, the $\mathrm{SOH}$ estimation precision will be higher when the sensor of voltage and current is more accurate.

\section{SOP Estimation Algorithm}

Battery SOP is defined the maximum charging or discharging power for a predetermined time interval, usually $10 \mathrm{~s}$ in studies [49]. To attain estimation of SOP, the peak current should be firstly taken into account. As well known, the real-time peak current of a battery is limited by its voltage, current, temperature, available capacity and SOC and is influenced by the electrochemical dynamics and thermodynamics inside of the battery. From (3) and (4), it can be found that SOC is an integration function of input current. To 
decouple the relationship and solve the current capability, the Taylor-series expansion is applied to linearize (3), as:

$$
f_{o c v}\left(s_{k}+\eta i_{k} \Delta t / Q_{a}\right)=f_{o c v}\left(s_{k}\right)+\left.i_{k+1} \frac{\eta i_{k} \Delta t}{C_{a}} \frac{\partial f_{o c v}}{\partial s}\right|_{s=s_{k}}+\Re
$$

Since the value of $\eta i_{k} \Delta t / Q_{a}$ is quite small, and the first-order residual $\mathfrak{R}$ can be as approximated to $\Re \approx 0$ [34], we can get:

$$
v_{t, k+1} \approx f_{o c v}\left(s_{k}\right)+\left.i_{k+1} \frac{\eta i_{k} \Delta t}{Q_{a}} \frac{\partial f_{o c v}}{\partial s}\right|_{s=s_{k}}+v_{1, k+1}+v_{2, k+1}+R_{0} i_{k+1}
$$

By incorporating (1) and (2), equation (21) can be rewritten as:

$$
\begin{aligned}
v_{t, k+1} & \approx f_{o c v}\left(s_{k}\right)+\left.i_{k+1} \frac{\eta i_{k} \Delta t}{Q_{a}} \frac{\partial f_{o c v}}{\partial s}\right|_{s=s_{k}}+a_{1, k} \cdot v_{1, k}+b_{1, k} i_{k+1} \\
& +a_{2, k} \cdot v_{2, k}+b_{2, k} i_{k+1}+R_{0} i_{k+1}
\end{aligned}
$$

The current at $k+1$ th step can be calculated as:

$$
i_{k+1}=\frac{v_{t, k+1}-f_{o c v}\left(s_{k}\right)-a_{1, k} \cdot v_{1, k}-a_{2, k} \cdot v_{2, k}}{\left.\frac{\eta i_{k} \Delta t}{Q_{a}} \frac{\partial f_{o c v}}{\partial s}\right|_{s=s_{k}}+b_{1, k}+b_{2, k}+R_{0}}
$$

Finally, by considering the operating voltage range, the maximum current from $t_{k}$ to $t_{k+1}$ can be attained:

$$
\left\{\begin{array}{c}
i_{k}^{d i s, v}=\frac{v_{t, \min }-f_{o c v}\left(s_{k}\right)-a_{1, k} \cdot v_{1, k}-a_{2, k} \cdot v_{2, k}}{\left.\frac{\eta i_{k} \Delta t}{Q_{a}} \frac{\partial f_{o c v}}{\partial s}\right|_{s=s_{k}}+b_{1, k}+b_{2, k}+R_{0}} \\
i_{k}^{c h a, v}=\frac{v_{t, \max }-f_{o c v}\left(s_{k}\right)-a_{1, k} \cdot v_{1, k}-a_{2, k} \cdot v_{2, k}}{\left.\frac{\eta i_{k} \Delta t}{Q_{a}} \frac{\partial f_{o c v}}{\partial s}\right|_{s=s_{k}}+b_{1, k}+b_{2, k}+R_{0}}
\end{array}\right.
$$

To operate safely when SOC decreases or increases to the designed thresholds, we should estimate the battery SOC and control the operating current lower than the maximum allowable value. The SOC based current capability estimates from $t_{k}$ to $t_{k+1}$ can be attained according to (3), as:

$$
\left\{\begin{array}{l}
i_{k}^{c h a, s}=\frac{s_{k}-s_{\max }}{\eta \Delta t / Q_{a}} \\
i_{k}^{d i s, s}=\frac{s_{k}-s_{\min }}{\eta \Delta t / Q_{a}}
\end{array}\right.
$$


where $s_{\min }$ and $s_{\max }$ represent the design limits of the lower and upper SOC values, respectively. On the other hand, the battery charging and discharging current should be subject to the manufacturer's specification, as illustrated in Table II.

Table II. The maximum permissible operating current.

\begin{tabular}{cccc}
\hline Temperature range $\left({ }^{\circ} \mathrm{C}\right)$ & {$[0,5]$} & $(5,15]$ & $(15,45)$ \\
charging & $0.1 \mathrm{C}$ & $0.2 \mathrm{C}$ & $0.5 \mathrm{C}$ \\
\hline Temperature range $\left({ }^{\circ} \mathrm{C}\right)$ & {$[-20,5]$} & $(5,45]$ & $(45,60]$ \\
discharging & $1 \mathrm{C}$ & $3 \mathrm{C}$ & $1.5 \mathrm{C}$ \\
\hline
\end{tabular}

Note: $\mathrm{C}$ denotes the battery rated capacity value with the unit of Ah.

By incorporating the discussed restrictions, the current capability from $t_{k}$ to $t_{k+1}$ can be formulated as:

$$
\left\{\begin{array}{l}
\hat{i}_{k}^{d i s}=\min \left\{i^{d i s, m}, i_{k}^{d i s, v}, i_{k}^{d i s, s}\right\} \\
\hat{i}_{k}^{c h a}=\max \left\{i^{c h a, m}, i_{k}^{c h a, v}, i_{k}^{c h a, s}\right\}
\end{array}\right.
$$

where $i^{d i s, m}$ and $i^{c h a, m}$ represent maximum discharging and charging current provided by the manufacturer's specification, respectively. Equation (26) gives the calculation method of current capability within the unit sampling interval. However, for EVs, duration of the peak current is critical, as acceleration, hill climbing, and braking energy recovery cannot be accomplished in single sampling duration. Thus, by considering the duration time $l$, and assuming the system's input current between step $t_{k}$ and step $t_{k+l}$ as a constant value, the state and measurement equations at step $k+l$ can be formulated, as:

$$
\left\{\begin{array}{l}
X_{k+l}=A_{k+l} \cdot X_{k+l-1}+B_{k+l} u_{k+l-1} \\
Y_{k+l}=C_{k+l} \cdot X_{k+l}+D_{k+l}
\end{array}\right.
$$

Supposing that the battery prediction model within the duration of $l$ steps is considered as a steady state system for simple calculation, the system states can be calculated, as:

$$
X_{k+l}=A_{k}^{l} X_{k}+\left(\sum_{j=0}^{l-1} A_{k}^{l-1-j} B_{k}\right) u_{k}
$$

Combining (2) and (28), the polarization voltage prediction model can be updated, as:

$$
\left\{\begin{array}{l}
v_{1, k+l}=a_{1, k}^{l} v_{1, k}+i_{k} \sum_{j=0}^{l-1} a_{1, k}^{l-1-j} b_{1, k} \\
v_{2, k+l}=a_{2, k}^{l} v_{1, k}+i_{k} \sum_{j=0}^{l-1} a_{2, k}^{l-1-j} b_{2, k}
\end{array}\right.
$$

Given this, the predicted terminal voltage can be yielded, as: 


$$
\begin{aligned}
v_{t, k+l} & =f_{\text {ocv }}\left(s_{k+l}\right)+\left[a_{1, k}^{l} v_{1, k}+i_{k} \sum_{j=0}^{l-1} a_{1, k}^{l-1-j} b_{1, k}\right]+\left[a_{2, k}^{l} v_{1, k}+i_{k} \sum_{j=0}^{l-1} a_{2, k}^{l-1-j} b_{2, k}\right]+i_{k} R_{0} \\
& =f_{o c v}\left(s_{k}+\frac{\eta i_{k+1} l \Delta t}{Q_{a}}\right)+\left[a_{1, k}^{l} v_{1, k}+i_{k} \sum_{j=0}^{l-1} a_{1, k}^{l-1-j} b_{1, k}\right]+\left[a_{2, k}^{l} v_{2, k}+i_{k} \sum_{j=0}^{l-1} a_{2, k}^{l-1-j} b_{2, k}\right]+i_{k} R_{0} \\
& =f_{o c v}\left(s_{k}\right)+\left.i_{k+l} \frac{\eta l \Delta t}{Q_{a}} \frac{\partial f_{o c v}}{\partial s}\right|_{s=s_{0}}+\left[a_{1, k}^{l} v_{1, k}+i_{k} \sum_{j=0}^{l-1} a_{1, k}^{l-1-j} b_{1, k}\right]+\left[a_{2, k}^{l} v_{2, k}+i_{k} \sum_{j=0}^{l-1} a_{2, k}^{l-1-j} b_{2, k}\right]+i_{k} R_{0}
\end{aligned}
$$

By solving (30), the voltage based maximum discharge current can be calculated, as:

$$
i_{k+l}=\frac{v_{t, k+l}-f_{o c v}\left(s_{k}\right)-a_{1, k}^{l}-a_{2, k}^{l} v_{2, k}}{\left.\frac{\eta l \Delta t}{Q_{a}} \frac{\partial f}{\partial s}\right|_{s=s_{k}}+\sum_{j=0}^{l-1} a_{1, k}^{l-1-j} b_{1, k}+i_{k} \sum_{j=0}^{l-1} a_{2, k}^{l-1-j} b_{2, k}+R_{.0}}
$$

Similarly, the voltage based continuous current capability can be determined, as:

$$
\left\{\begin{array}{l}
i_{k+l}^{d i s, v}=\frac{v_{t, \min }-f_{o c v}\left(s_{k}\right)-a_{1, k}^{l} v_{1, k}-a_{2, k}^{l} v_{2, k}}{\left.\frac{\eta l \Delta t}{Q_{a}} \frac{\partial f_{o c v}}{\partial s}\right|_{s=s_{k}}+\sum_{j=0}^{l-1} a_{1, k}^{l-1-j} b_{1, k}+i_{k} \sum_{j=0}^{l-1} a_{2, k}^{l-1-j} b_{2, k}+R_{0}} \\
i_{k+l}^{c h a, v}=\frac{v_{t, \max }-f_{o c v}\left(s_{k}\right)-a_{1, k}^{l} v_{1, k}-a_{2, k}^{l} v_{2, k}}{\left.\frac{\eta l \Delta t}{Q_{a}} \frac{\partial f_{o c v}}{\partial s}\right|_{s=s_{k}}+\sum_{j=0}^{l-1} a_{1, k}^{l-1-j} b_{1, k}+i_{k} \sum_{j=0}^{l-1} a_{2, k}^{l-1-j} b_{2, k}+R_{0}}
\end{array}\right.
$$

Furthermore, the SOC based continuous current capability can be calculated, as:

$$
\left\{\begin{array}{l}
i_{k+l}^{c h a, s}=\frac{s_{k}-s_{\max }}{\ln \Delta t / Q_{a}} \\
i_{k+l}^{d i s, s}=\frac{s_{k}-s_{\min }}{\ln \Delta t / Q_{a}}
\end{array}\right.
$$

By combining the battery SOC based, terminal voltage based and manufacturer based restrictions, the multiconstraint continuous current capability can be formulated as:

$$
\left\{\begin{array}{l}
\hat{i}_{k+l}^{d i s}=\max \left\{i^{d i s, m}, i_{k+l}^{d i s, v}, i_{k+l}^{d i s, s}\right\} \\
\hat{i}_{k+l}^{c h a}=\min \left\{i^{c h a, m}, i_{k+l}^{c h a, v}, i_{k+l}^{c h a, s}\right\}
\end{array}\right.
$$

Finally, the SOP can be attained when the terminal voltage prediction model is considered, as:

$$
\left\{\begin{array}{l}
P_{k+l}^{d i s}=\hat{i}_{k+l}^{d i s} v_{t, k+l} \\
P_{k+l}^{c h a}=\hat{i}_{k+l}^{c h a} v_{t, k+l}
\end{array}\right.
$$

By this manner, the SOP can be finally determined with the full consideration of different constraints. After designing the inner status co-estimation algorithm, a series of experiments, including offline test and 
real BMS validation, are conducted to comprehensively verify the feasibility, effectiveness and robustness of proposed algorithm.

\section{OFFLINE VALIDATION AND DisCUSSION}

In this section, several vital factors are considered in the offline validation, including the current and voltage noises, ambient temperature variation, and varying characteristics of battery aging status. To comprehensively validate the algorithm, different experiments were conducted, and the results are discussed, including the SOC estimation with existence of measurement noise, the algorithm's robustness under different temperatures, and the effectiveness with battery degradation.

\section{A. SOC Validation with Current and Voltage Noise}

To validate the filtering effect of proposed SOC estimation algorithm, the battery is discharged at room temperature under the urban dynamometer driving schedule (UDDS) experiment. The Gaussian noise, of which the mean and variance equal 0 and $0.5 \mathrm{~A}^{2}$, is overlapped into the measured current and the portion current profiles are shown in Fig. 4 (a). Meanwhile, the zero-mean Gaussian noise with variance of $0.01 \mathrm{~V}^{2}$ is imposed on the measured voltage, as depicted in Fig. 4 (b). The proposed AEKF-FF, AEKF and conventional EKF are sequentially implemented to estimate the SOC under dynamic load conditions. Fig. 4 (c) and (d) compare the SOC estimation results, and the errors based on different algorithms are summarized in Table III. The initial values of SOC are all set to $90 \%$ with $10 \%$ initial error. It can be found that the AEKF-FF, AEKF and EKF can all track the reference SOC quickly. The proposed AEKF-FF estimator can raise more accurate estimation, compared to the AEKF and EKF algorithms; and the mean error (ME) and root mean square error (RMSE) based on the proposed AEKF-FF are respectively reduced to $0.45 \%$ and $0.23 \%$ when the process and measurement noise is not imposed. In contrast, when the noise is added to the original measured current, the SOC estimation error based on the EKF algorithm obviously rises; nonetheless, the error by the AEKF-FF and AEKF only increases slightly. For the AEKF-FF, the RMSE, ME and maximum error are respectively $0.48 \%, 0.19 \%$ and $0.74 \%$, only half of those by the AEKF and one third of those by the EKF. Thus, the experimental validation manifests that the proposed AEKF-FF algorithm is more 
effective to eliminate the noise and attain precise SOC estimation, compared to traditional AEKF and EKF algorithms.

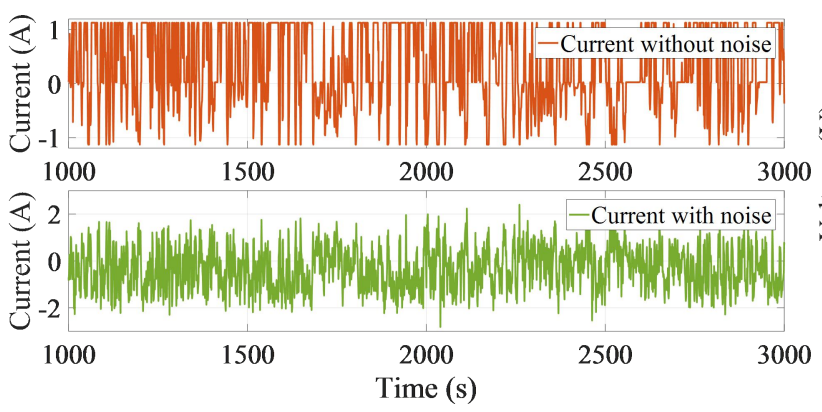

(a)

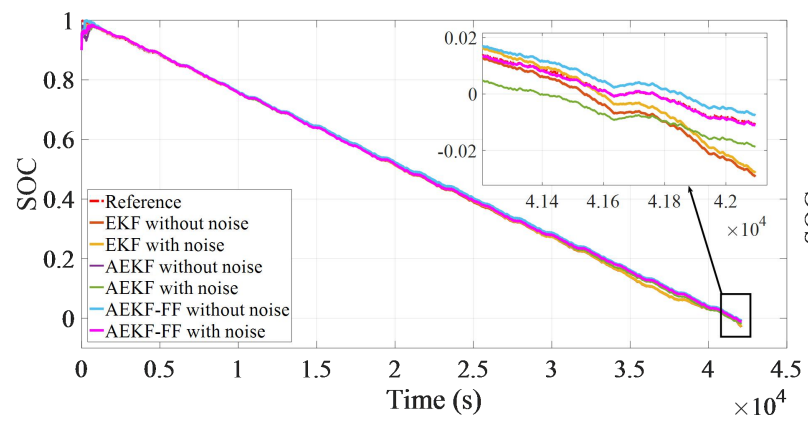

(c)

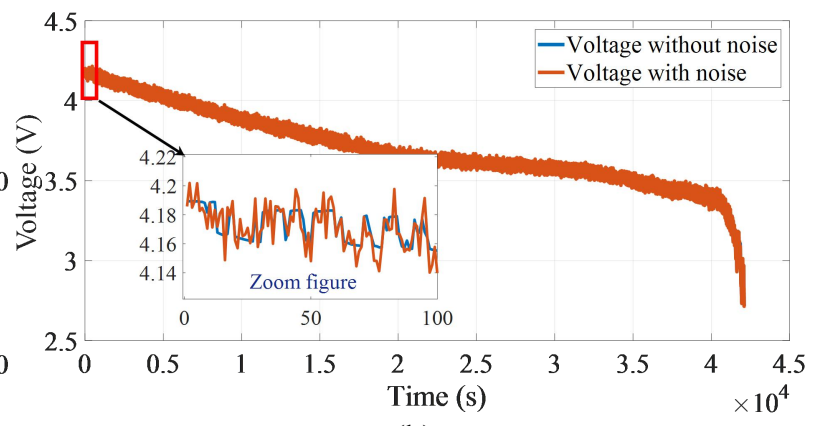

(b)

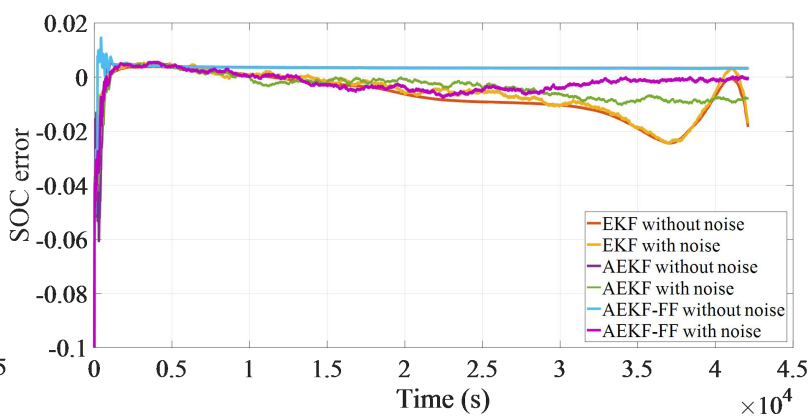

(d)

Fig. 4. Comparison between measured and noisy. (a) Measured and noisy current; (b) Measured and noisy voltage; (c) SOC estimation result; (d) SOC estimation error.

Table III. Comparison of SOC estimation results.

\begin{tabular}{lllll}
\hline Algorithm & Noise & RMSE (\%) & Maximum error (\%) & ME (\%) \\
\hline \multirow{2}{*}{ EKF } & Without noise & 1.00 & 2.43 & 0.64 \\
& With noise & 1.33 & 3.05 & 0.86 \\
\multirow{2}{*}{ AEKF } & Without noise & 0.59 & 0.51 & 0.29 \\
& With noise & 0.81 & 1.15 & 0.50 \\
\multirow{2}{*}{ AEKF-FF } & Without noise & 0.45 & 0.37 & 0.23 \\
& With noise & 0.48 & 0.74 & 0.19 \\
\hline
\end{tabular}

\section{B. SOC and SOP Validation at Different Temperatures}

The battery performance highly relies on the operating temperature, and the model parameters also correlates with the temperature. Wide variation of operating temperature will influence estimation accuracy and robustness of SOC. Additionally, similar phenomenon also appears at high temperature. Thus, it is obligatory to assess the performance of presented co-estimation algorithm under different temperature conditions.

First, the battery is discharged under UDDS cycle; and then fully charged with $0.5 \mathrm{C}$ current. Next, the battery is discharged under the dynamic stress test (DST) cycle until the voltage reaches $2.75 \mathrm{~V}$. All the experiments are carried out at $55^{\circ} \mathrm{C}$. The measured current and voltage profiles are plotted in Fig. 5 (a) and (b). The estimated SOC in terms of different algorithms is depicted in Fig. 5 (c), and Fig. 5 (d) describes the 
SOC estimation errors. Note that the reference SOC is obtained at the same temperature according to the SOC definition, and the SOC estimation results without the temperature correction are yielded from the identified parameters at $25{ }^{\circ} \mathrm{C}$. The initial SOC (actually $100 \%$ ) is set to $80 \%$ when conducting estimations. From Fig. 5 and Table IV, it can be observed that at $55^{\circ} \mathrm{C}$, the RMSE is $2.69 \%$ when the traditional AEKF algorithm is implemented without temperature compensation. Similarly, the estimation error by the Ah counting method rises to a large extent due to the capacity variation, and the RMSE based on the Ah integration method also exceeds $1.4 \%$. For the proposed AEKF-FF algorithm and AEKF algorithm with temperature correction, the RMSE is restricted within $1.09 \%$ and $1.4 \%$, highlighting the effectiveness of AEKF-FF in SOC estimation at high temperature.

Table IV. Comparison of SOC estimation results at $55^{\circ} \mathrm{C}$.

\begin{tabular}{lllll}
\hline Algorithm & Temperature & Maximum error (\%) & RMSE (\%) & ME (\%) \\
\hline Ah & Without temperature correction & 2.92 & 1.41 & 1.18 \\
\multirow{2}{*}{ AEKF } & Without temperature correction & 4.40 & 2.69 & 2.07 \\
& With temperature correction & 3.60 & 1.40 & 1.12 \\
\multirow{2}{*}{ AEKF-FF } & Without temperature correction & 4.90 & 2.86 & 2.21 \\
& With temperature correction & 3.20 & 1.09 & 0.80 \\
\hline
\end{tabular}

Fig. 5 (e) to (h) show the current capability and SOP variation at $55^{\circ} \mathrm{C}$, and the minimum SOC is set to $2 \%$. It can be found that the estimated SOP are influenced by two aspects. The discharge SOP is subject to the manufacturer specifications when the SOC remains in the higher range, and the SOP is determined by the SOC based restriction when the SOC approximates to the minimum limit. On the other hand, since the charge operation is not allowed at $55{ }^{\circ} \mathrm{C}$, the SOP in the charging condition is zero subject to multi constraints according to the specification. Fig. 5 (h) shows the SOP variation when the minimum SOC is set to $2.3 \%$. It can be clearly observed that when the SOC declines to $2.3 \%$, the allowable SOP reaches zero simultaneously, indicating the viability of proposed algorithm.

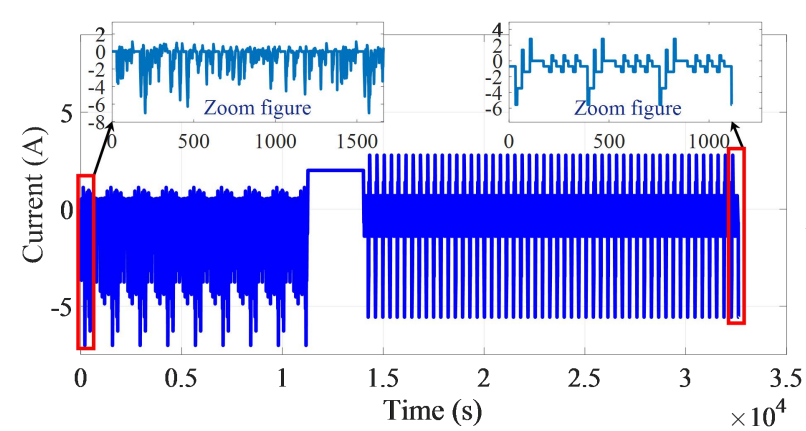

(a)

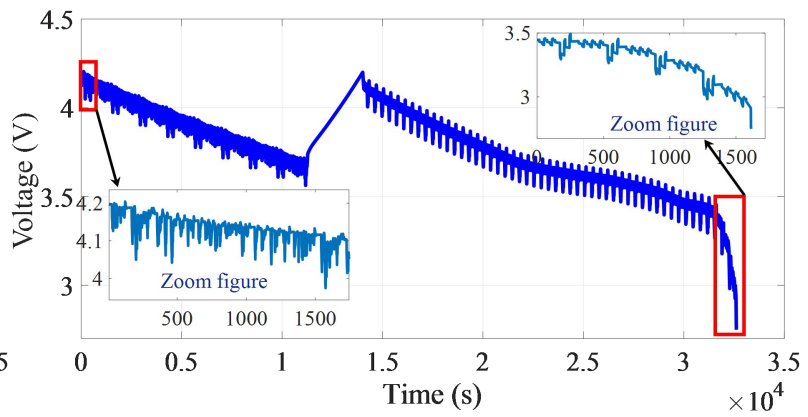

(b) 


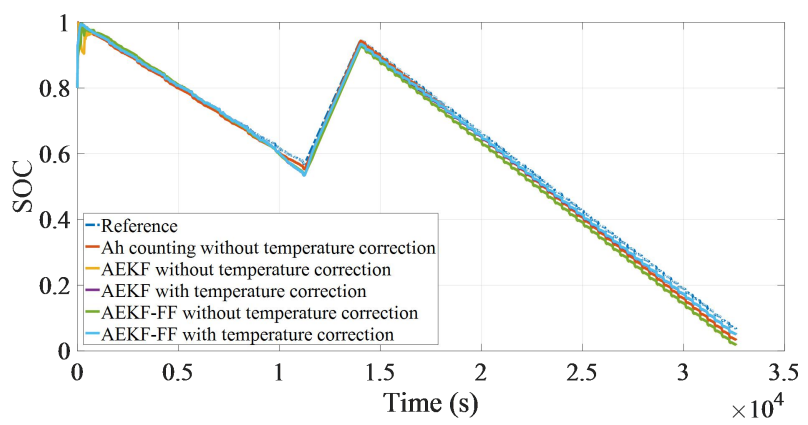

(c)

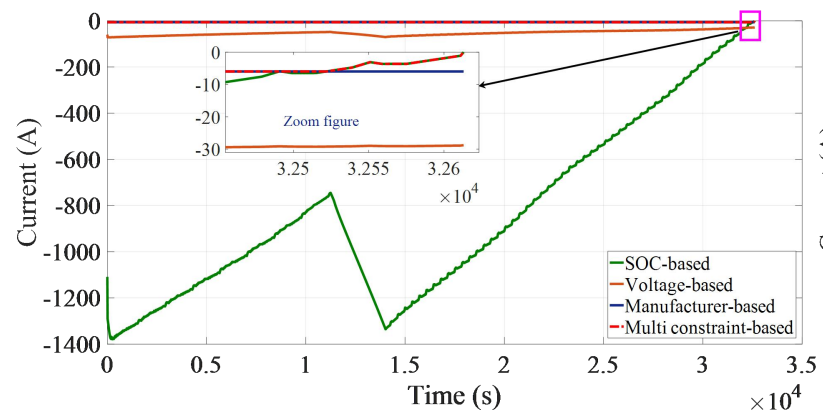

(e)

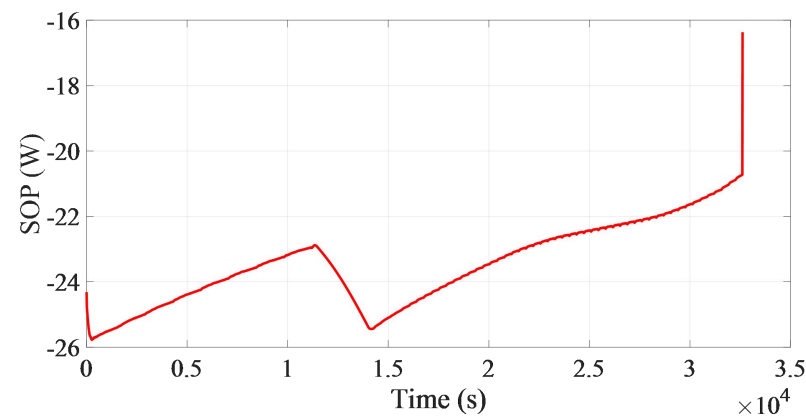

(g)

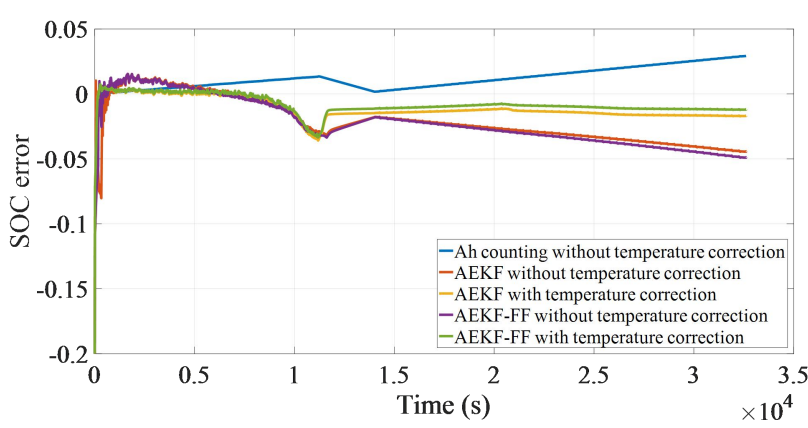

(d)

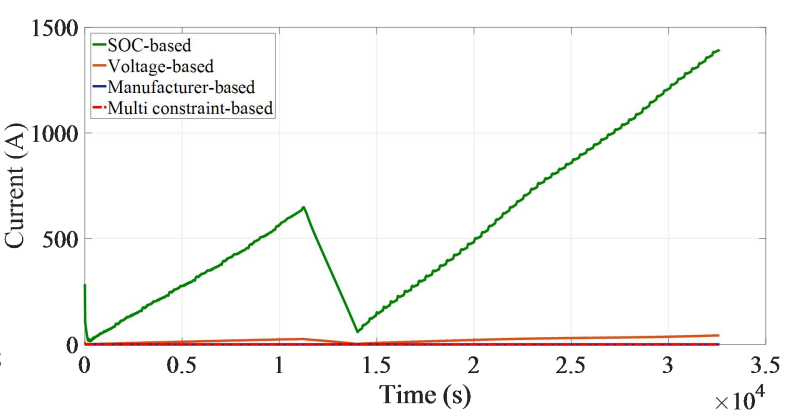

(f)

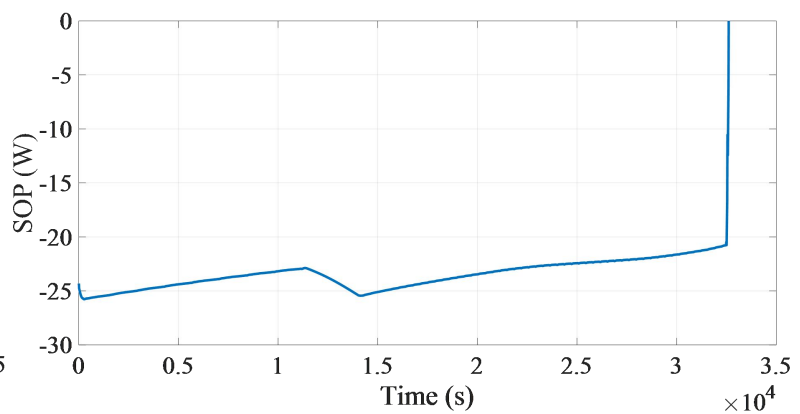

(h)

Fig. 5. SOC and SOP estimation results at $55^{\circ} \mathrm{C}$. (a) Hybrid current profiles; (b) Voltage profiles; (c) SOC reference and estimation results; (d) SOC estimation error; (e) Discharging current capability with $s_{\min }=2 \%$; (f) Charging current capability; (g) Multi-constraint SOP; (h) Discharging SOP with $s_{\min }=2.3 \%$.

In addition, more experiments are conduct at varying temperatures to further validate the proposed temperature compensation based SOC and SOP. In the test, the battery is discharged under federal urban driving schedule (FUDS) cycle test, followed by the full charge with constant current (CC)-constant voltage (CC-CV) scheme. Finally, the battery is discharged under the FUDS cycle until the voltage reaches $2.75 \mathrm{~V}$. In this experiment, the temperature rises from $4{ }^{\circ} \mathrm{C}$ to $53{ }^{\circ} \mathrm{C}$ by gradually heating the battery in a thermal controlled chamber. The measured current, voltage and temperature are plotted in Fig. 6 (a) to (c).

To examine the convergence of co-estimation algorithm in terms of abrupt initial SOC, different initial SOC values $(60 \%$ and $30 \%)$ are set in the proposed algorithm, in contrast to the actual value of $100 \%$. The comparison results of online SOC estimation are discussed in Fig. 6 (d) and (e). As can be seen, the SOC 
estimation error based on the improved AEKF-FF algorithm with temperature correction is confined within $1.2 \%$ error in the entire experiment. The AEKF-FF without the temperature correction shows the worst performance, and the maximum error is more than 3.5\%. Fig. 6 (f) to (i) illustrates the co-estimation results for the current capability and SOP. From these figures, we can find that when the battery is operated under the varying temperature conditions, the SOP changes in accordance with the operating temperature. When the SOC is close to its high threshold (100\% in this study), the charging SOP becomes quite small while the discharging SOP is large; thereby effectively avoiding overcharge. On the contrary, when the SOC approximates to the lower limit, the charging SOP becomes large, whereas the discharging SOP turns to be small. In this manner, the over-discharge can be avoided. To sum up, the proposed algorithm can improve the estimation accuracy of SOC, compared with traditional AEKF/EKF algorithms. Furthermore, the SOC can also be accurately estimated when the operating temperature changes dramatically. In addition, the proposed temperature correction-based SOP algorithm can adapt to temperature variation and also improve operation safety in the case of unknown initial SOC.

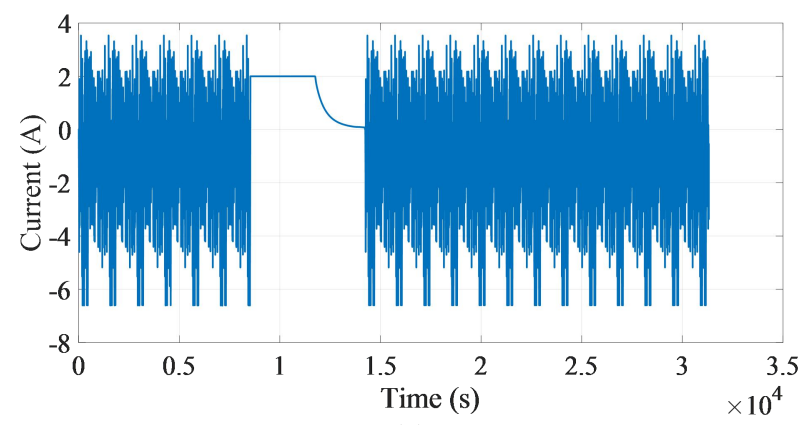

(a)

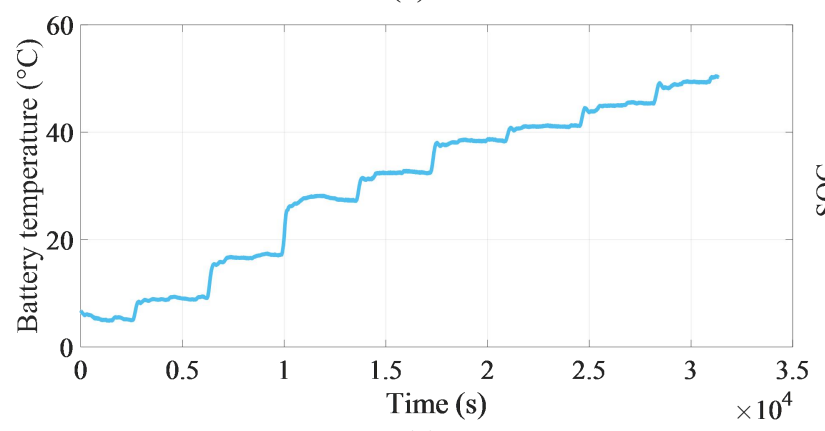

(c)

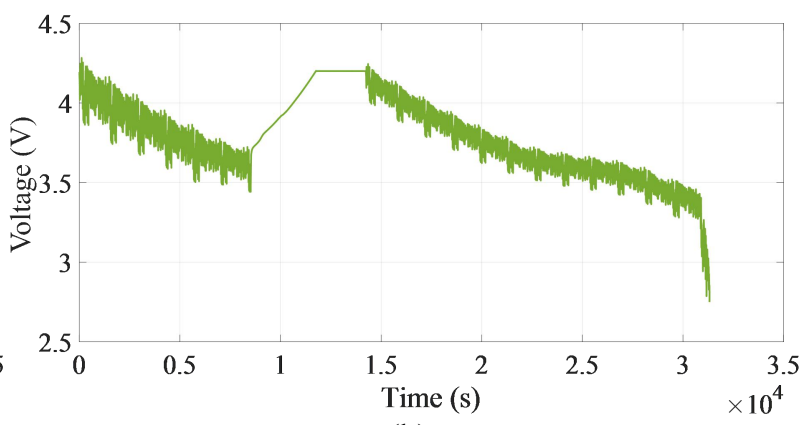

(b)

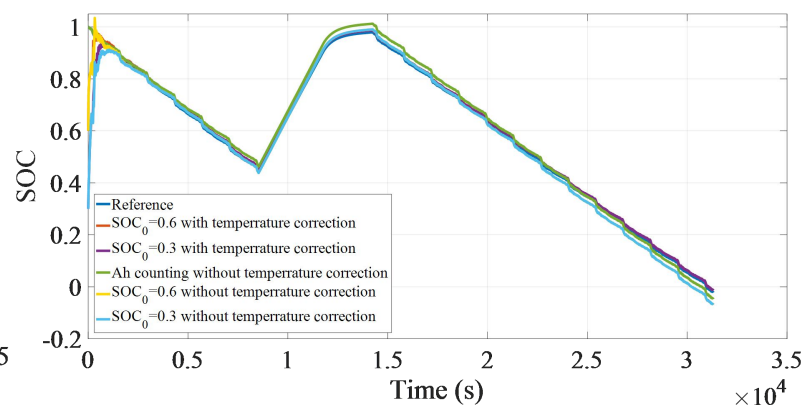

(d) 


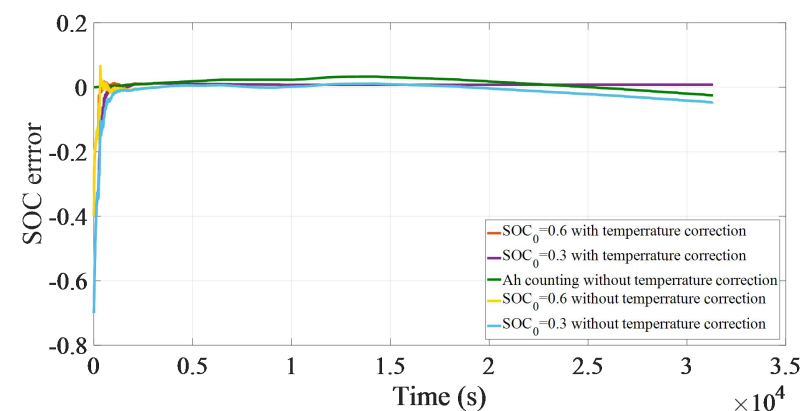

(e)

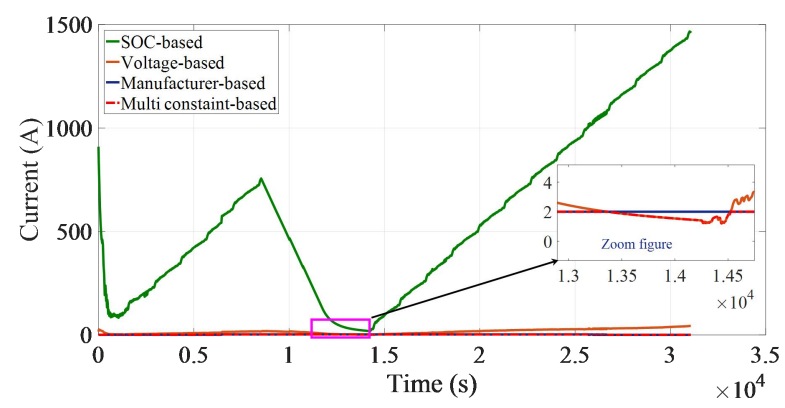

(g)

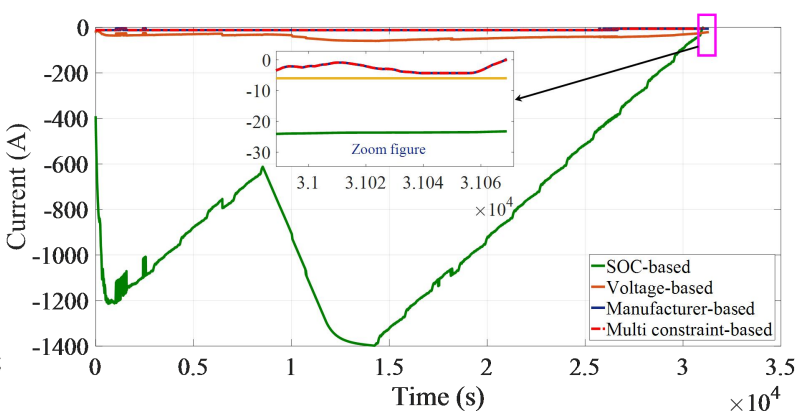

(f)

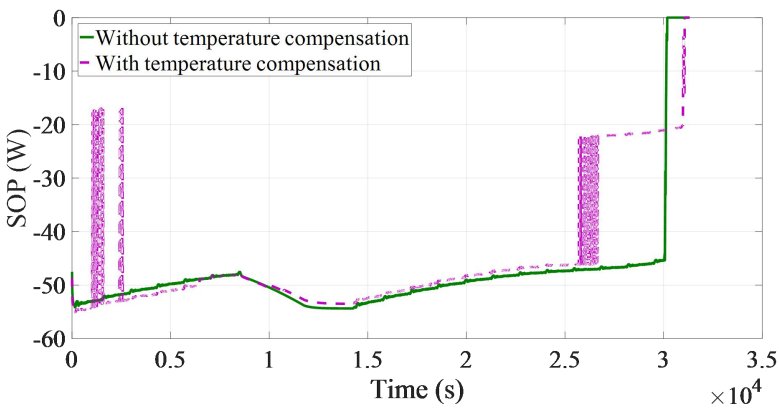

(h)

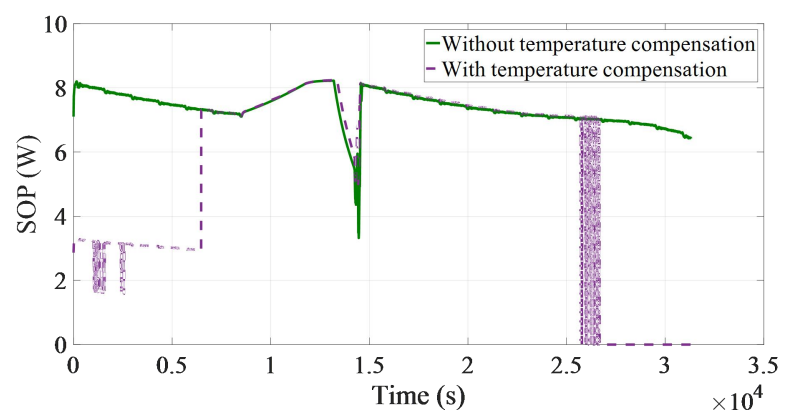

(i)

Fig. 6. Measurement data and estimation results at dynamic temperature. (a) Hybrid current profiles; (b) Voltage response profiles; (c) Battery temperature variation profiles; (d) SOC curves; (e) SOC estimation error; (f) Discharging current capability; (g) Charging current capability; (h) Multi-constraint discharging SOP; (i) Multi-constraint charging SOP.

\section{Validation for Aged Cells}

To examine whether the presented algorithm has a promising application against different degradation status, the aged cells are tested to verify the co-estimation algorithm. The battery aging test is performed with repeated discharging and charging process. At each cycle, the battery is charged with the standard CC-CV strategy, the $\mathrm{CC}$ charging current is $0.5 \mathrm{C}$, the cut-off current is $0.02 \mathrm{C}$, and the $\mathrm{CC}$ discharging current is $2 \mathrm{C}$. The charging and discharging cut-off voltage of the life test battery are set as $4.2 \mathrm{~V}$ and $2.75 \mathrm{~V}$, respectively. The HPPC test is conducted after a period of cycle experiments. When the battery is shelved more than $1 \mathrm{~h}$ and the rest voltage is higher than the feature voltage, the $\mathrm{SOH}$ calculation is trigger according to the proposed algorithm. Here, five battery cells that meet the SOH update criteria are employed to validate the 
proposed $\mathrm{SOH}$ estimation algorithm. The capacity and $\mathrm{SOH}$ estimation results are shown in Fig. 7 (a) and Table V. We can find that the maximum absolute error of capacity is $0.16 \mathrm{Ah}$, and the maximum absolute error of $\mathrm{SOH}$ is $4.13 \%$. Furthermore, we can find the estimation accuracy of $\mathrm{SOH}$ gradually improves as the cycle experiment goes on. The SOH estimation error can be always limited within $3 \%$ before the SOH drops to $80 \%$, thus verifying the feasibility of proposed $\mathrm{SOH}$ estimation algorithm.

Table V. SOH estimation results.

\begin{tabular}{lllllll}
\hline $\begin{array}{l}\text { Cell } \\
\text { num. }\end{array}$ & $\begin{array}{l}\text { Reference } \\
\text { SOH (\%) }\end{array}$ & $\begin{array}{l}\text { Rest } \\
(\mathrm{V})\end{array}$ & $\begin{array}{l}\text { voltage } \\
\text { table (\%) }\end{array}$ & $\begin{array}{l}\text { SOC by look-up } \\
\text { capacity (Ah) }\end{array}$ & $\begin{array}{l}\text { Estimation } \\
\text { SOH (\%) }\end{array}$ & Error (\%) \\
\hline 1 & 93.71 & 3.8345 & 70.2 & 1.13 & 97.84 & 4.13 \\
2 & 91.02 & 3.8308 & 69.7 & 1.10 & 93.68 & 2.66 \\
3 & 87.84 & 3.8327 & 70.0 & 1.06 & 91.17 & 3.33 \\
4 & 85.18 & 3.8295 & 69.6 & 1.03 & 87.42 & 2.24 \\
5 & 81.75 & 3.8250 & 69.1 & 0.99 & 82.67 & 0.92 \\
\hline
\end{tabular}

After attaining the battery SOH, the SOC is estimated based on the HPPC data. In the validation case, the $\mathrm{SOH}$ is $81.75 \%$, and the SOC estimation results are illustrated in Fig. 7 (b). The reference SOC is estimated by the Ah integration method with the updated capacity and the $80 \%$ initial SOC is given in the proposed SOC estimator. The absolute error of estimation results is depicted in Fig. 7 (c), showing that the estimation error based on the proposed estimator can be restricted within $2 \%$. This broadly proved the effectiveness of proposed SOC estimation algorithm even in the aged battery.

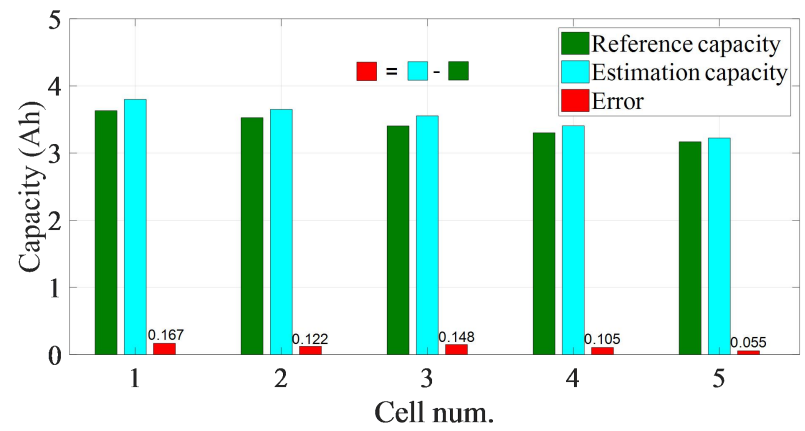

(a)

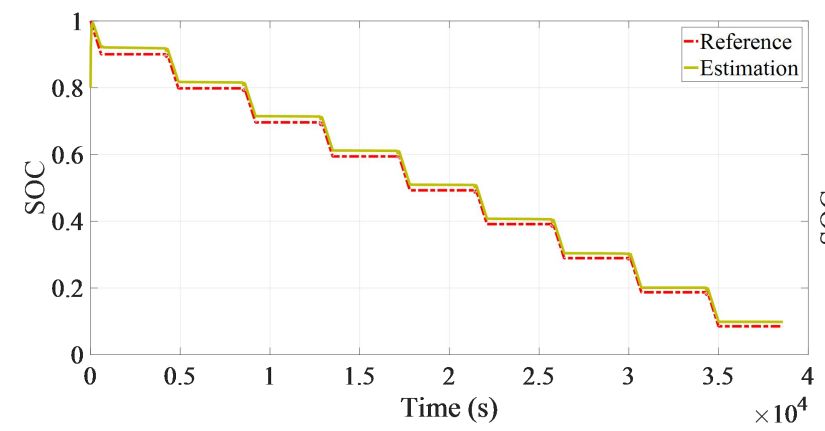

(b)

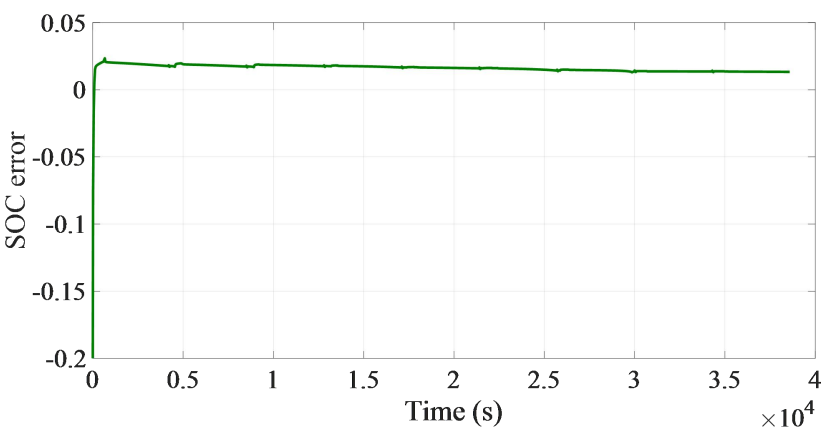

(c)

Fig. 7. Estimation results for an aging cell. (a) Capacity estimation results; (b) SOC curves; (c) Absolute error. 


\section{Algorithm Validation in A Production Battery PaCK}

To further validate the practicability of proposed multi-state co-estimator, a real BMS testbed is set up, as shown in Fig. 8 (a). It consists of a battery pack with six cells connected in parallel and fourteen cells connected in series, a BMS circuit, a direct current (DC) electronic load, a programmable temperature chamber, a laptop computer with controller area network (CAN) test software to save data, and a laptop computer with host computer software to display battery pack parameters. The BMS accounts for acquiring the real-time data including pack current, cell voltage and temperature and estimating the inner status of each parallel-connected cell. The accuracy of current transducer is $2 \%$, and the measure error of cell voltage is less than $15 \mathrm{mV}$.

The measured current and cell voltage are plotted in Fig. 8 (b) to (d). Due to the communication noise, the current curve shows some sharp noise. Similar with the current profiles, the voltage is not as smooth as the offline test data. Fig. 8 (e) shows the temperature variation of battery pack, it can be found that the battery temperature changes from $28{ }^{\circ} \mathrm{C}$ to $33{ }^{\circ} \mathrm{C}$. The initial SOC of tested cells are $100 \%$ and the initial SOC values in the BMS are incorrectly set to $20 \%$. Obviously, the initial error is $80 \%$. Fig. 8 (f) to (i) provides the estimation results. As can be seen, the maximum SOC estimation error is less than $1.5 \%$ after convergence. Based on the estimated SOC, all the cells' discharge SOP is estimated online, as described in Fig. 8 (h), and the discharge SOP of battery pack is shown in Fig. 8 (i). It can be found that the discharge SOP of battery pack is determined by the minimum discharging current of battery cell. In addition, the SOC estimation error is less than $1.5 \%$ owning to online update of model parameters corresponding to temperature variation. According to the validation results, the proposed algorithm is proved feasible in the product BMS with preferable real-time performance. 


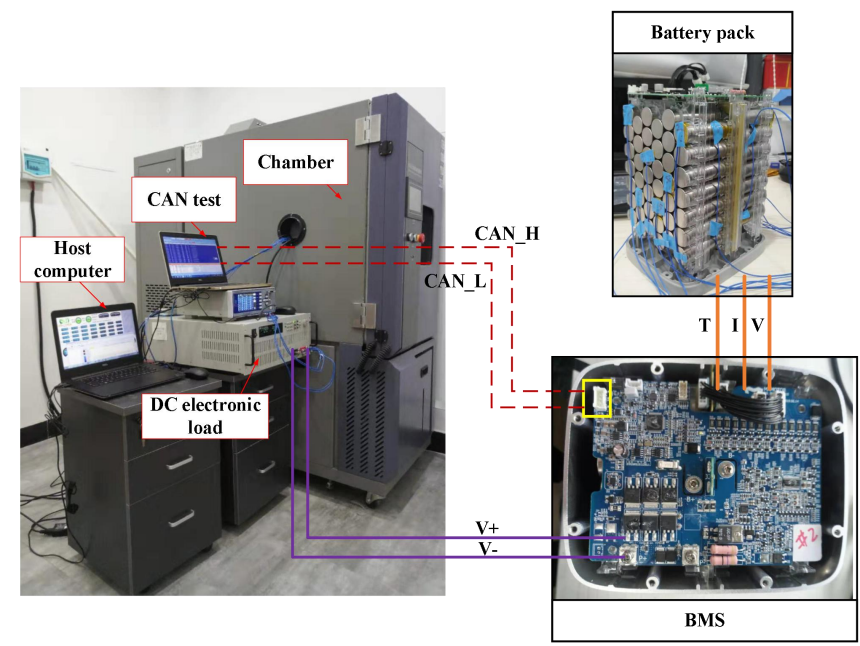

(a)

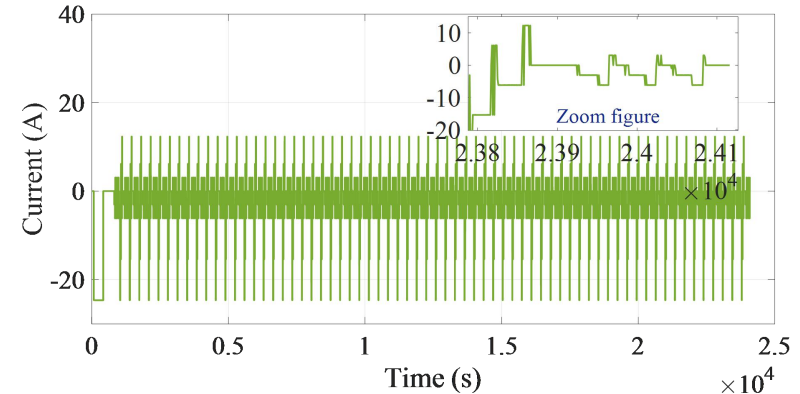

(b)

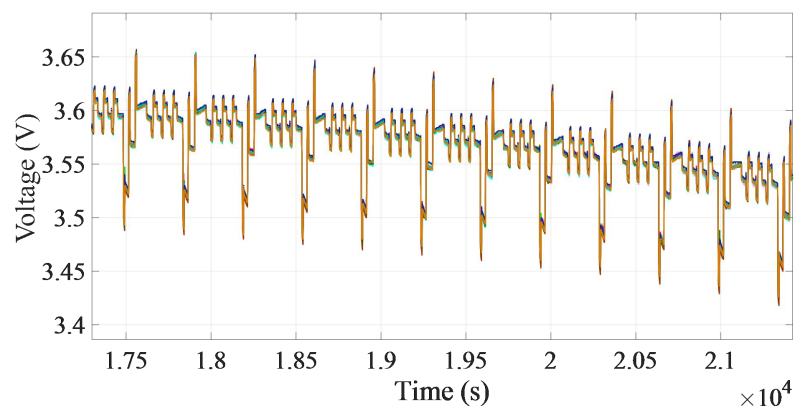

(d)

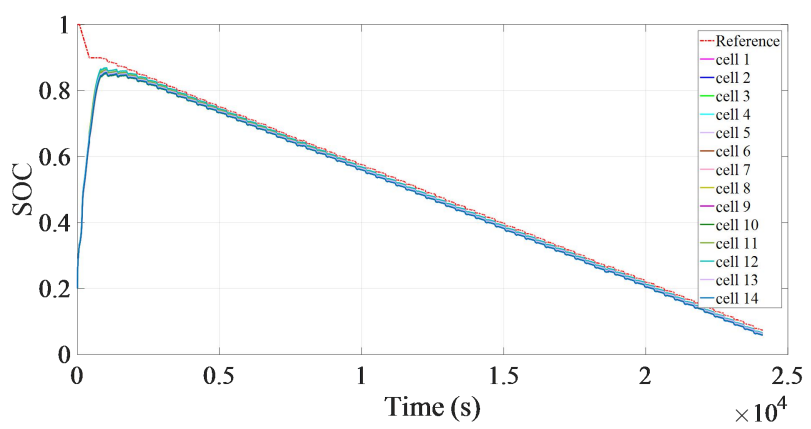

(f)

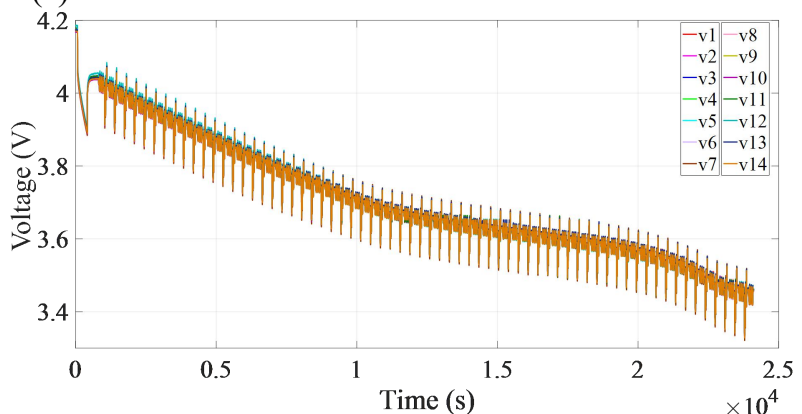

(c)

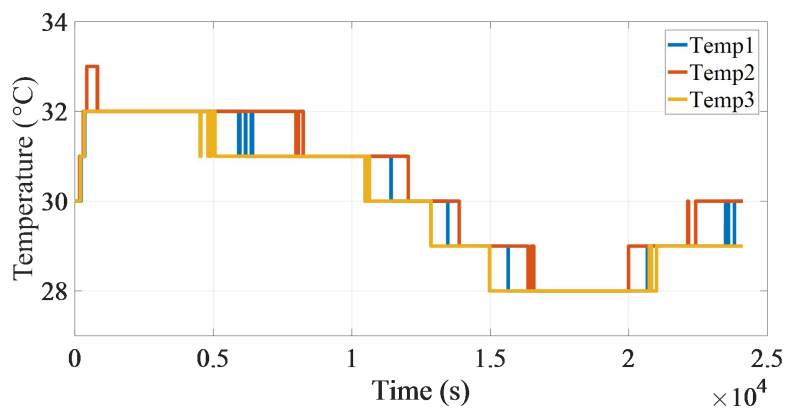

(e)

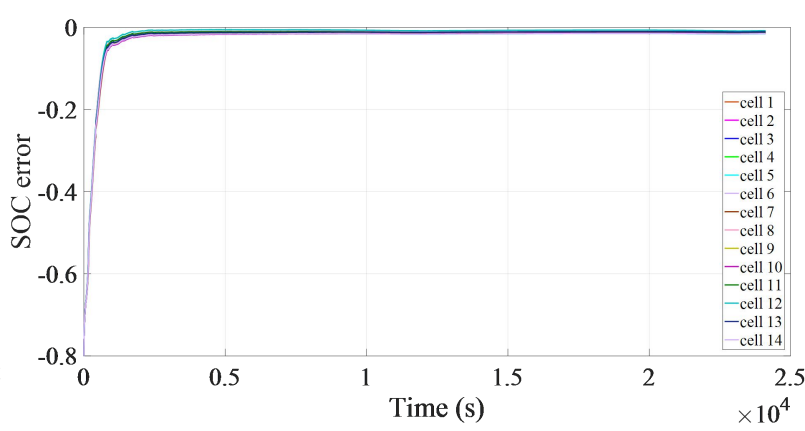

(g) 


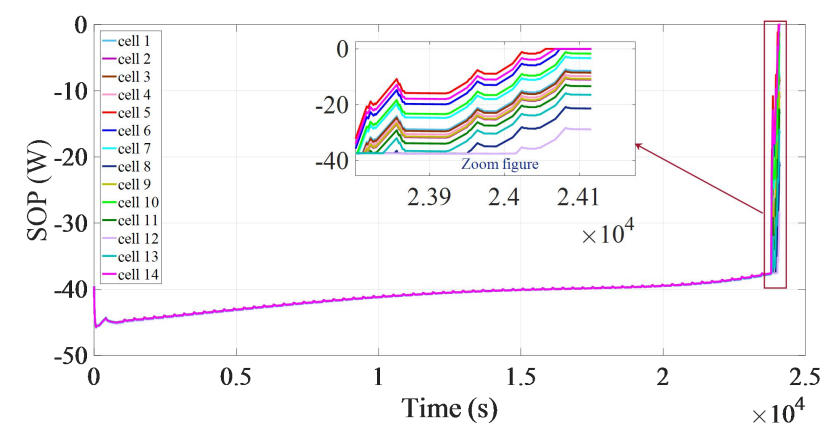

(h)

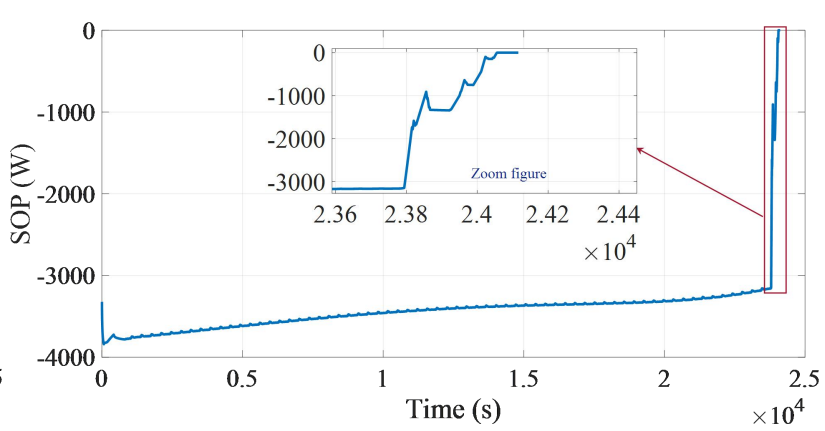

(i)

Fig. 8. The built testing platform and algorithm validation results in real BMS. (a) Testing platform; (b) Current profiles; (c) Battery cell voltage profiles; (d) Enlarged cell voltage profiles; (e) Battery temperature profiles; (f) SOC estimation curves; (g) SOC estimation errors; (h) Discharging SOP of each cell; (i) Discharging SOP of battery pack.

\section{CONCLUSIONS}

In this paper, an adaptive multi-state estimation algorithm is proposed for the battery cell and pack with the consideration of temperature compensation. The state of charge estimation is achieved based on the second-order equivalent circuit model and the adaptive extended Kalman filter with a forgetting factor. To ensure the charging and discharging safety of battery, the battery state of power estimation is properly tackled by incorporating the constraints from voltage, current, state of charge and temperature. The state of health estimation is conducted by the open circuit voltage-based feature point method. Compared with the traditional extended Kalman filter and adaptive extended Kalman filter, the experimental results highlight the following four main findings:

1) The adaptive extended Kalman filter with a forgetting factor method is employed to reliably estimate state of charge of batteries thanks to the update of historical information. The proposed algorithm attains more accurate estimation of state of charge, compared to the traditional adaptive extended Kalman filter and ampere hour methods, under varying temperature conditions due to the timely model compensation.

2) The proposed algorithm provides the authentic estimation of state of power by incorporating the multi constraints and duration of maximum current.

3) The proposed fusion algorithm can estimate the battery $\mathrm{SOH}$ with the maximum error of $4.13 \%$. On the basis of accurate state of health, the adaptive extended Kalman filter with a forgetting factor can maintain the state of charge estimation error with $2 \%$ even in the aged battery. 
4) The proposed co-estimator is confirmed effective in engineering applications where fast calculation and accurate inner states estimation entail.

The future work will focus on influences of cell inconsistency on the state of charge and maximum available energy of the battery pack under different temperatures.

\section{ACKNOWLEDGEMENTS}

This work was supported in part by the National Natural Science Foundation (No. 51775063 and No. 61763021), in part by the National Key R\&D Program of China (No. 2018YFB0104900), in part by Chongqing Fundamental Research and Frontier Exploration Project (No. CSTC2019JCYJ-MSXMX0642), and in part by the EU-funded Marie Skłodowska-Curie Individual Fellowships Project under Grant 845102HOEMEV-H2020-MSCA-IF-2018. In addition, the authors would like to thank the Shenzhen Mamotor Technology Co., Ltd. for their hardware and program training support. Moreover, and most importantly, the authors would like to express deep gratitude to the anonymous reviewers for their corrections and helpful suggestions.

\section{REFERENCES}

[1] C. Zhang, F. Yang, X. Ke, Z. Liu, and C. Yuan, "Predictive modeling of energy consumption and greenhouse gas emissions from autonomous electric vehicle operations," Applied Energy, vol. 254, p. 113597, 2019.

[2] K. Liu, K. Li, Q. Peng, and C. Zhang, "A brief review on key technologies in the battery management system of electric vehicles," Frontiers of Mechanical Engineering, vol. 14, pp. 47-64, 2019.

[3] X. Shu, G. Li, J. Shen, W. Yan, Z. Chen, and Y. Liu, "An adaptive fusion estimation algorithm for state of charge of lithium-ion batteries considering wide operating temperature and degradation," Journal of Power Sources, vol. 462, p. 228132, 2020.

[4] J. Yang, B. Xia, Y. Shang, W. Huang, and C.C. Mi, "Adaptive State-of-Charge Estimation Based on a Split Battery Model for Electric Vehicle Applications," IEEE Transactions on Vehicular Technology, vol. 66, pp. 10889-10898, 2017.

[5] M.A. Hannan, M.H. Lipu, A. Hussain, and A. Mohamed, "A review of lithium-ion battery state of charge estimation and management system in electric vehicle applications: Challenges and recommendations," Renewable and Sustainable Energy Reviews, vol. 78, pp. 834-854, 2017.

[6] L. Zheng, J. Zhu, G. Wang, D.D.-C. Lu, and T. He, "Differential voltage analysis based state of charge estimation methods for lithium-ion batteries using extended Kalman filter and particle filter," Energy, vol. 158, pp. 1028-1037, 2018.

[7] W. Wang and J. Mu, "State of Charge Estimation for Lithium-Ion Battery in Electric Vehicle Based on Kalman Filter Considering Model Error," IEEE Access, vol. 7, pp. 29223-29235, 2019.

[8] C. Chen, R. Xiong, R. Yang, W. Shen, and F. Sun, "State-of-charge estimation of lithium-ion battery using an improved neural network model and extended Kalman filter," Journal of Cleaner Production, vol. 234, pp. 1153-1164, 2019. 
[9] Y. Zheng, W. Gao, M. Ouyang, L. Lu, L. Zhou, and X. Han, "State-of-charge inconsistency estimation of lithium-ion battery pack using mean-difference model and extended Kalman filter," Journal of Power Sources, vol. 383, pp. 50-58, 2018.

[10] S.-L. Wang, C. Fernandez, W. Cao, C.-Y. Zou, C.-M. Yu, and X.-X. Li, "An adaptive working state iterative calculation method of the power battery by using the improved Kalman filtering algorithm and considering the relaxation effect," Journal of Power Sources, vol. 428, pp. 67-75, 2019.

[11] Y. Li, J. Chen, and F. Lan, "Enhanced online model identification and state of charge estimation for lithiumion battery under noise corrupted measurements by bias compensation recursive least squares," Journal of Power Sources, vol. 456, p. 227984, 2020.

[12] R. Xiong, L. Li, Q. Yu, Q. Jin, and R. Yang, "A set membership theory based parameter and state of charge co-estimation method for all-climate batteries," Journal of Cleaner Production, vol. 249, p. 119380, 2020.

[13] Y. Bi and S.-Y. Choe, "An adaptive sigma-point Kalman filter with state equality constraints for online stateof-charge estimation of a $\mathrm{Li}(\mathrm{NiMnCo}) \mathrm{O} 2 / \mathrm{Carbon}$ battery using a reduced-order electrochemical model," Applied Energy, vol. 258, p. 113925, 2020.

[14] H. Sheng and J. Xiao, "Electric vehicle state of charge estimation: Nonlinear correlation and fuzzy support vector machine," Journal of Power Sources, vol. 281, pp. 131-137, 2015.

[15] Y. Liu, J. Li, Z. Chen, D. Qin, and Y. Zhang, "Research on a multi-objective hierarchical prediction energy management strategy for range extended fuel cell vehicles," Journal of Power Sources, vol. 429, pp. 55-66, 2019.

[16] J. Du, Z. Liu, and Y. Wang, "State of charge estimation for Li-ion battery based on model from extreme learning machine," Control Engineering Practice, vol. 26, pp. 11-19, 2014.

[17] R. Xiong, J. Cao, Q. Yu, H. He, and F. Sun, "Critical Review on the Battery State of Charge Estimation Methods for Electric Vehicles," IEEE Access, vol. 6, pp. 1832-1843, 2018.

[18] F. Yang, W. Li, C. Li, and Q. Miao, "State-of-charge estimation of lithium-ion batteries based on gated recurrent neural network," Energy, vol. 175, pp. 66-75, 2019.

[19] Y. Tian, R. Lai, X. Li, L. Xiang, and J. Tian, "A combined method for state-of-charge estimation for lithiumion batteries using a long short-term memory network and an adaptive cubature Kalman filter," Applied Energy, vol. 265, p. 114789, 2020.

[20] D. Yang, X. Zhang, R. Pan, Y. Wang, and Z. Chen, "A novel Gaussian process regression model for state-ofhealth estimation of lithium-ion battery using charging curve," Journal of Power Sources, vol. 384, pp. 387395, 2018.

[21] P. Guo, Z. Cheng, and L. Yang, "A data-driven remaining capacity estimation approach for lithium-ion batteries based on charging health feature extraction," Journal of Power Sources, vol. 412, pp. 442-450, 2019.

[22] D. Yang, Y. Wang, R. Pan, R. Chen, and Z. Chen, "State-of-health estimation for the lithium-ion battery based on support vector regression," Applied Energy, vol. 227, pp. 273-283, 2018.

[23] J. Kim, S. Lee, and B. Cho, "Complementary cooperation algorithm based on DEKF combined with pattern recognition for SOC/capacity estimation and SOH prediction," IEEE Transactions on Power Electronics, vol. 27, pp. 436-451, 2011.

[24] D. Liu, X. Yin, Y. Song, W. Liu, and Y. Peng, "An on-line state of health estimation of lithium-ion battery using unscented particle filter," Ieee Access, vol. 6, pp. 40990-41001, 2018.

[25] M.H. Lipu, M. Hannan, A. Hussain, M. Hoque, P.J. Ker, M.H.M. Saad, and A. Ayob, "A review of state of health and remaining useful life estimation methods for lithium-ion battery in electric vehicles: Challenges and recommendations," Journal of Cleaner Production, vol. 205, pp. 115-133, 2018.

[26] H. Pan, Z. Lü, H. Wang, H. Wei, and L. Chen, "Novel battery state-of-health online estimation method using multiple health indicators and an extreme learning machine," Energy, vol. 160, pp. 466-477, 2018.

[27] S.S.Y. Ng, Y. Xing, and K.L. Tsui, "A naive Bayes model for robust remaining useful life prediction of lithium-ion battery," Applied Energy, vol. 118, pp. 114-123, 2014.

[28] Y. Deng, H. Ying, E. Jiaqiang, H. Zhu, K. Wei, J. Chen, et al., "Feature parameter extraction and intelligent estimation of the State-of-Health of lithium-ion batteries," Energy, vol. 176, pp. 91-102, 2019.

[29] K. Liu, X. Hu, Z. Wei, Y. Li, and Y. Jiang, "Modified Gaussian Process Regression Models for Cyclic Capacity Prediction of Lithium-ion Batteries," IEEE Transactions on Transportation Electrification, 2019.

[30] Z. Zhou, Y. Kang, Y. Shang, N. Cui, C. Zhang, and B. Duan, "Peak power prediction for series-connected LiNCM battery pack based on representative cells," Journal of Cleaner Production, vol. 230, pp. 1061-1073, 2019.

[31] Z. Wei, J. Zhao, R. Xiong, G. Dong, J. Pou, and K.J. Tseng, "Online estimation of power capacity with noise effect attenuation for lithium-ion battery," IEEE Transactions on Industrial Electronics, vol. 66, pp. 5724-5735, 2018.

[32] J.R. Belt, "Battery test manual for plug-in hybrid electric vehicles," Idaho National Laboratory (INL)2010. 
[33] G.L. Plett, "High-performance battery-pack power estimation using a dynamic cell model," IEEE Transactions on vehicular technology, vol. 53, pp. 1586-1593, 2004.

[34] R. Xiong, F. Sun, H. He, and T.D. Nguyen, "A data-driven adaptive state of charge and power capability joint estimator of lithium-ion polymer battery used in electric vehicles," Energy, vol. 63, pp. 295-308, 2013.

[35] L. Yang, Y. Cai, Y. Yang, and Z. Deng, "Supervisory long-term prediction of state of available power for lithium-ion batteries in electric vehicles," Applied Energy, vol. 257, p. 114006, 2020.

[36] Z. Wei, J. Zhao, R. Xiong, G. Dong, J. Pou, and K. J. Tseng, "Online estimation of power capacity with noise effect attenuation for lithium-ion battery," IEEE Transactions on Industrial Electronics, vol. 66, pp. 5724-5735, 2018.

[37] P. Shen, M. Ouyang, L. Lu, J. Li, and X. Feng, "The Co-estimation of State of Charge, State of Health, and State of Function for Lithium-Ion Batteries in Electric Vehicles," IEEE Transactions on Vehicular Technology, vol. 67, pp. 92-103, 2018.

[38] H. Chaoui and C.C. Ibe-Ekeocha, "State of charge and state of health estimation for lithium batteries using recurrent neural networks," IEEE Transactions on vehicular technology, vol. 66, pp. 8773-8783, 2017.

[39] X. Li, Z. Wang, and L. Zhang, "Co-estimation of capacity and state-of-charge for lithium-ion batteries in electric vehicles," Energy, vol. 174, pp. 33-44, 2019.

[40] X. Zhang, Y. Wang, C. Liu, and Z. Chen, "A novel approach of remaining discharge energy prediction for large format lithium-ion battery pack," Journal of Power Sources, vol. 343, pp. 216-225, 2017.

[41] L. Zheng, J. Zhu, D.D.-C. Lu, G. Wang, and T. He, "Incremental capacity analysis and differential voltage analysis based state of charge and capacity estimation for lithium-ion batteries," Energy, vol. 150, pp. 759-769, 2018.

[42] X. Hu, H. Jiang, F. Feng, and B. Liu, "An enhanced multi-state estimation hierarchy for advanced lithium-ion battery management," Applied Energy, vol. 257, p. 114019, 2020.

[43] W.D. Widanage, A. Barai, G.H. Chouchelamane, K. Uddin, A. McGordon, J. Marco, and P. Jennings, "Design and use of multisine signals for Li-ion battery equivalent circuit modelling. Part 2: Model estimation," Journal of Power Sources, vol. 324, pp. 61-69, 2016.

[44] Z. Chen, X. Shu, R. Xiao, W. Yan, Y. Liu, and J. Shen, "Optimal charging strategy design for lithium-ion batteries considering minimization of temperature rise and energy loss," International Journal of Energy Research, vol. 0, 2019.

[45] C. Huang, Z. Wang, Z. Zhao, L. Wang, C.S. Lai, and D. Wang, "Robustness Evaluation of Extended and Unscented Kalman Filter for Battery State of Charge Estimation," IEEE Access, vol. 6, pp. 27617-27628, 2018.

[46] Z. Liu, X. Dang, and B. Jing, "A Novel Open Circuit Voltage Based State of Charge Estimation for LithiumIon Battery by Multi-Innovation Kalman Filter," IEEE Access, vol. 7, pp. 49432-49447, 2019.

[47] L. Lu, X. Han, J. Li, J. Hua, and M. Ouyang, "A review on the key issues for lithium-ion battery management in electric vehicles," Journal of Power Sources, vol. 226, pp. 272-288, 2013.

[48] C. Hu, B.D. Youn, and J. Chung, "A multiscale framework with extended Kalman filter for lithium-ion battery SOC and capacity estimation," Applied Energy, vol. 92, pp. 694-704, 2012.

[49] W. Waag, C. Fleischer, and D.U. Sauer, "Adaptive on-line prediction of the available power of lithium-ion batteries," Journal of Power Sources, vol. 242, pp. 548-559, 2013. 